\title{
Combinatorial epigenetic mechanisms and efficacy of early breast cancer inhibition by nutritive botanicals
}

\begin{abstract}
Aim: Aberrant epigenetic events are important contributors to the pathogenesis of different types of cancers and dietary botanicals with epigenetic properties can influence early cancer development leading to cancer prevention effects. We sought to investigate potential combinatorial effects of bioactive dietary components including green tea polyphenols (GTPs) and broccoli sprouts (BSp) on neutralizing epigenetic aberrations during breast tumorigenesis. Materials \& methods: The combinatorial effects were evaluated in a breast cancer transformation cellular system and breast cancer mouse xenografts. Results \& conclusion: Combined treatment with epigallocatechin-3-gallate in GTPs and sulforaphane in BSp resulted in a synergistic inhibition of breast cancer cellular growth. Further studies revealed this combination led to genome-wide epigenetic alterations. Combinatorial diets significantly inhibited tumor growth in breast cancer mouse xenografts. Collectively, these studies indicate that combined GTPs and BSp are highly effective in inhibiting early breast cancer development by, at least in part, regulating epigenetic mechanisms.
\end{abstract}

First draft submitted: 3 March 2016; Accepted for publication: 19 May 2016; Published online: 1 August 2016

Keywords: breast tumorigenesis $\bullet$ broccoli sprouts $\bullet$ EGCG $\bullet$ epigenetic $\bullet$ green tea polyphenols • sulforaphane

Breast cancer is the most common cancer and the second leading cause of cancer death among women [1]. Although the precise mechanisms involved in breast tumorigenesis are still under intense investigation, it has been recently widely accepted that epigenetic aberration-induced gene expression changes play a major role in initiating breast carcinogenic processes [2-4]. Epigenetic mechanisms control gene expression via changing the accessibility of chromatin to transcriptional regulation locally and globally through modifications of the DNA and modification or rearrangement of nucleosomes [5-7]. Two major epigenetic mechanisms, DNA methylation and histone acetylation/methylation, are essential for organismal development and regulation of tissue-specific gene expression during normal physiological pro- cesses in mammals. However, disruption of epigenetic processes can lead to altered gene function and malignant cellular transformation $[2,5,8]$. It has become increasingly apparent that epigenetic alterations frequently occur in neoplastic cells involving abnormal epigenetic patterns in DNA methylation and histone modifications as well as dysregulaglobal changes in the epigenetic landscape are a hallmark of cancer. Recently, numerous drugs that target epigenetic pathways have emerged and been widely tested in different clinical trials targeting various cancer types [9,10]. However, high toxicity due to lack of specific targets in neoplastic cells as well as relatively low efficacy for solid tumors have largely limited epigenetic drugs applied in clinical practice for cancer therapy. Thus, tion of epigenetic mechanisms. Therefore,
Epigenomics

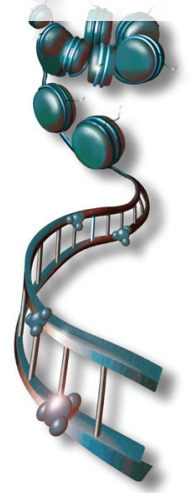

Yuanyuan Li $i^{*, 1,2,3}$, Phillip Buckhaults $^{4}$, Xiangqin Cui ${ }^{5}$ \& Trygve O Tollefsbol $1,2,3,6$

'Department of Biology, University of Alabama at Birmingham, Birmingham, AL 35294, USA

${ }^{2}$ Comprehensive Cancer Center, University of Alabama at Birmingham, Birmingham, AL 35294, USA ${ }^{3}$ Nutrition Obesity Research Center, University of Alabama at Birmingham, Birmingham, AL 35294, USA

${ }^{4}$ Department of Drug Discovery \& Biomedical Sciences, University of South Carolina, Columbia, SC 29208, USA

${ }^{5}$ Department of Biostatistics, University of Alabama at Birmingham, Birmingham, AL 35294, USA

${ }^{6}$ Comprehensive Center for Healthy Aging, University of Alabama at Birmingham, Birmingham, AL 35294, USA *Author for correspondence:

Tel.: +1 2059344587

Fax: +1 2059756097

lyy@uab.edu 
exploration of less toxic, more attainable and effective natural compounds has been an emerging approach of epigenetic cancer therapy.

The 'epigenetic diets' refer to a small portion of bioactive diets with potent anticancer properties regulated via epigenetic events. Green tea polyphenols (GTPs) and broccoli sprouts (BSp) are two important epigenetic botanicals that have been extensively studied in our laboratory [11-15]. Epigallocatechin-3-gallate (EGCG) is a major polyphenol in green tea that has attracted intense interest. Studies have shown that green tea EGCG exhibits robust anticancer properties both in vitro and in vivo through regulation of multiple mechanisms including the induction of cell cycle arrest and apoptosis as well as inhibition of tumor metastasis and angiogenesis $[16,17]$. Sulforaphane (SFN) is an isothiocyanate from cruciferous vegetables such as BSp and cabbage that has been well documented to reduce the risk of developing many common cancers through several mechanisms including cell cycle arrest, induction of apoptosis and Phase II detoxification enzymes [18,19]. Interest in epigenetic regulation by EGCG and SFN in chemoprevention has recently surged due to their DNMTs and HDACs inhibition activities which lead to global and local alterations of DNA methylation and histone acetylation status of a number of tumorrelated genes that may reverse tumor progression processes [20,21]. Although our previous studies show potent effects of EGCG and SFN in preventing breast cancer when administered singly, it is important to test combinatorial effects of these two compounds that may overcome limitations of efficacy when acting alone and enhance safe and efficacious doses for consumption.

In the present study, we analyzed potential epigenetic mechanisms of combinatorial treatment with GTP/EGCG and BSp/SFN and their chemopreventive effects in a novel breast cancer cellular model, which resembles the processes of pathological progression and molecular events during earlier breast tumorigenesis. We observed that the combination of these botanicals resulted in a synergistic inhibition of cellular growth in precancerous breast cells and early breast cancer cells via, at least in part, regulating epigenetic mechanisms. This study will facilitate more effective uses of combinatorial epigenetic dietary approaches in breast cancer prevention and therapy.

\section{Materials \& methods}

\section{Cell culture \& cell treatment}

Normal human mammary epithelial cells (HMECs) were purchased from Lonza (Basel, Switzerland) at 15-20 population doublings. HMECs cells were stably transfected with either $S V 40$ and $h T E R T$ to obtain estrogen receptor (ER)-negative early transformed pre- cancerous cells referred to $\mathrm{SH}$ cells, or additional oncogene $H$-Ras to obtain completely transformed breast cancer referred to SHR cells as done previously in our laboratory [22,23]. HMECs cells were grown in serumfree mammary epithelial growth medium (MEGM) accompanied with MEGM SingleQuots (Lonza). Precancerous $\mathrm{SH}$ and early transformed breast cancer SHR cells were grown in Dulbecco's Modified Eagle Medium (Invitrogen, CA, USA) supplemented with $10 \%$ fetal bovine serum (Atlanta Biologicals, Lawrenceville, GA, USA) and 1\% penicillin/streptomycin (Mediatech, VA, USA). Culture cells were maintained in a humidified environment of $5 \% \mathrm{CO}_{2}$ and $95 \%$ air at $37^{\circ} \mathrm{C}$ and treated with indicted concentration of EGCG and/or SFN to evaluate the combinatorial effect of EGCG and SFN (Sigma, MO, USA) treatment. The culture medium was replaced every $24 \mathrm{~h}$ for the duration of the experiment.

\section{MTT assay for cell viability}

Aliquots of cells were seeded in triplicate in 96-well plates and treated with the indicated concentrations of EGCG and/or SFN to determine the effects of combinatorial treatment on cell viability. The MTT reagent (Sigma) was added to the culture medium followed by 4-h incubation at $37^{\circ} \mathrm{C}$ until purple precipitates are visible. The media were aspirated and the cells were dissolved in $100 \mu \mathrm{l}$ DMSO. The absorbance of the cell lysates was measured at $570 \mathrm{~nm}$ by a microtiter plate reader (Bio-Rad, CA, USA) as done previously [23].

\section{Cell apoptosis \& cell cycle analysis}

Precancerous $\mathrm{SH}$ cells and early transformed breast cancer SHR cells treated with either EGCG at $20 \mu \mathrm{M}$ or SFN at $10 \mu \mathrm{M}$ alone or together were collected and washed with cold phosphate-buffered saline (PBS). Cellular apoptosis was analyzed by the Vybrant Apoptosis Assay kit \#2 (Invitrogen) as reported previously [23]. PI staining-based flow cytometry cell cycle assay was used to analyze cell cycle distribution. After washing with PBS, cells were fixed in $70 \%$ ethanol at $-20^{\circ} \mathrm{C}$ overnight and washed with PBS twice. Cells were then suspended in PBS containing 0.1\% Triton X-100, $0.1 \%$ RNase and $50 \mu \mathrm{g} / \mathrm{ml}$ PI and incubated in dark for about $30 \mathrm{~min}$. Flow cytometry was used for both cell apoptosis and cell cycle analyses on a Becton Dickinson FACSCalibur Flow Cytometer. The fluorescence intensity of the viable cells was analyzed using CellQuest software.

\section{Quantitative real-time PCR}

Both precancerous SH and early transformed breast cancer SHR cells were cultured and treated as described above. Total RNAs from cells or mice tumor tissues 
were extracted using the RNeasy kit (Qiagen, CA, USA) according to the manufacturer's instructions and reversely transcribed to cDNA using iScript cDNA Synthesis kit (Biorad) as done previously $[14,23]$. Gene expression were performed in triplicate and analyzed by real-time PCR using SYBR GreenER qPCR Supermix (Invitrogen) in a Roche LC480 thermocycler. Specific gene primers for DNMT1, HDAC1, DCBLD2, Septin 9 and GAPDH were provided by Integrated DNA Technologies as following: 5'-ACCGCTTCTACTTCCTCGAGGCCTA-3' (F), 5'-GTTGCAGTCCTCTGTGAACACTGTGG-3' (R) for DNMT1; 5'-ATGGACGATCTGTTTCCCCT-3' (F), 5'-CGGTTTACTCGGCAGATCTT-3' (R) for HDAC1; 5'-GGGTAGTTTATGGATGTAAGGGT-3' (F), 5'-CTGTTCCTCCTGCTCTTACTTG-3' (R) for DCBLD2; 5'-CACAGCAAATGGGATCATCTC-3' (F), 5'-CACTTCAAACAGCGGATCAC-3' (R) for Septin 9 and 5'-ACCACAGTCCATGCCATCAC-3' (F) and 5'TCCACCACCCTGTTGCTGTA-3' (R) for $G A P D H$. Thermal cycling was initiated at $94^{\circ} \mathrm{C}$ for 4 min followed by 30 cycles of three steps of PCR amplification processes $\left(94^{\circ} \mathrm{C}, 15 \mathrm{~s} ; 60^{\circ} \mathrm{C}, 30 \mathrm{~s}\right)$ and melting curve analysis. GAPDH was used as an internal control and vehicle control was used as a calibrator. The relative changes of gene expression in fold change were calculated using the following formula: $2^{-\Delta \Delta \mathrm{Ct}}=2^{-4 \mathrm{ACt}}$ (treated samples) $-\Delta \mathrm{Ct}$ (control samples) $\}$, where $\Delta \mathrm{Ct}=\mathrm{Ct}$ (test gene) - Ct (GAPDH) and Ct represents threshold cycle number.

\section{HDACs \& DNMTs activity assay}

Cultured cells were harvested at the indicated time points and nuclear proteins from SH and SHR cells were extracted. The activities of HDACs and DNMTs were evaluated by EpiQuik HDAC Activity/Inhibition Assay Kit and EpiQuik DNMT Activity/Inhibition Assay Kit (Epigentek, NY, USA) according to the manufacturer's protocols, respectively, as done previously [23].

\section{Global histone $\mathrm{H} 3$ acetylation assay}

Cultured SH and SHR cells were harvested by the indicated treatment of EGCG and/or SFN as described above. Histone protein was extracted and histone $\mathrm{H} 3$ acetylation level was detected according to the manufacturer's protocol of EpiQuik ${ }^{\mathrm{TM}}$ Global Histone H3 Acetylation Assay Kit (Epigentek). The histone H3 acetylation level was detected by using a microplate reader at $450 \mathrm{~nm}$.

\section{Genome-wide DNA methylation analysis}

Early transformed breast cancer SHR cells were cultured and treated as described above. Total DNA from SHR cells were extracted and converted using the EpiTect Bisulfite Kits (Qiagen) according to manufacturer instructions. Genome-wide DNA methylation status was measured at $485,577 \mathrm{CpG}$ islands using Illumina's Infinium HumanMethylation450 BeadChip [24]. Illumina’s GenomeStudio ${ }^{\circledR}$ methylation module was used to calculate the methylation level at each $\mathrm{CpG}$ island as the beta-value ( $\beta=$ intensity of the methylated allele $[\mathrm{M}] /[$ intensity of the unmethylated allele $(\mathrm{U})+$ intensity of the methylated allele $(M)+100])$. Bioconductor package minfi was used to process and analyze the BeadChip data. The data were normalized using the SWAN (subset-quantile within array normalization) method to reduce the variability between the two types of probes. Beta-values were then transformed to obtain the $\log$ ratio, defined as $\log (\beta /[1-\beta])$ to compare between the treatments.

\section{Western blot analysis}

Cultured SH and SHR cells were treated by the indicated treatments of EGCG and/or SFN and total proteins from the cells were extracted by RIPA Lysis Buffer (Upstate Biotechnology, VA, USA). Proteins were electrophoresed in Biorad SDS-polyacrylamide ready gels and transferred onto nitrocellulose membranes. Membranes were then probed with different antibodies to DCBLD2 (Thermo Scientific, Waltham, MA, USA), Septin 9 (Thermo Scientific, Pierce), HDAC1 (H11; Santa Cruz Biotechnology) and DNMT1 (ab 13537; Abcam, CA, USA) respectively, and each membrane was stripped and reprobed with beta-actin antibody (13E5, Cell Signaling Technology, MA, USA) as loading control. Immunoreactive bands were visualized using the enhanced chemiluminescence detection system (Santa Cruz Biotechnology) and documented by a ChemiDoc ${ }^{\mathrm{TM}}$ Imaging Systems (Biorad).

\section{RNA interference}

Validated siRNA for DNMT1 (ID: S4215) and HDAC1 (ID: S73) and the positive/negative control RNAi (Thermo Scientific, Applied Biosystems) were transfected into early transformed SHR cells by indicated treatment using the Silencer siRNA Transfection II Kit (Thermo Scientific) according to the protocols provided by the manufacturer [23].

Animal models, dietary \& experimental designs An orthotopic breast cancer mouse model was used in this study. Virgin female immunodeficiency $\mathrm{Nu} / \mathrm{Nu}$ Nude mice (Crl:NU-Foxn1nu) at 4-6 weeks of age were obtained from Charles River Laboratories (Wilmington, MA, USA) and housed in the Animal Resource Facility of the University of Alabama at Birmingham (UAB) under the following conditions: 12-h dark/12-h light cycle, $24 \pm 2^{\circ} \mathrm{C}$ temperatures and $50 \pm$ $10 \%$ humidity. An online power calculator [25] was used 
to calculate the power/sample size by two-proportion comparison based on our in vitro data. A sample size of five mice/group will give us $75 \%$ power for detecting beneficial botanical effects for a one-sided test at significance level of $0.05(\alpha=0.05)$.
After 1 week of acclimatization, $\mathrm{Nu} / \mathrm{Nu}$ Nude mice were randomly divided into four experimental groups (five mice each): control group: mice were fed with AIN-93G diet; GTPs diet: 0.5\% (5 mg/ml) of GTPs (Sunphenon 90D, Taiyo International, Inc., MN,

(A)

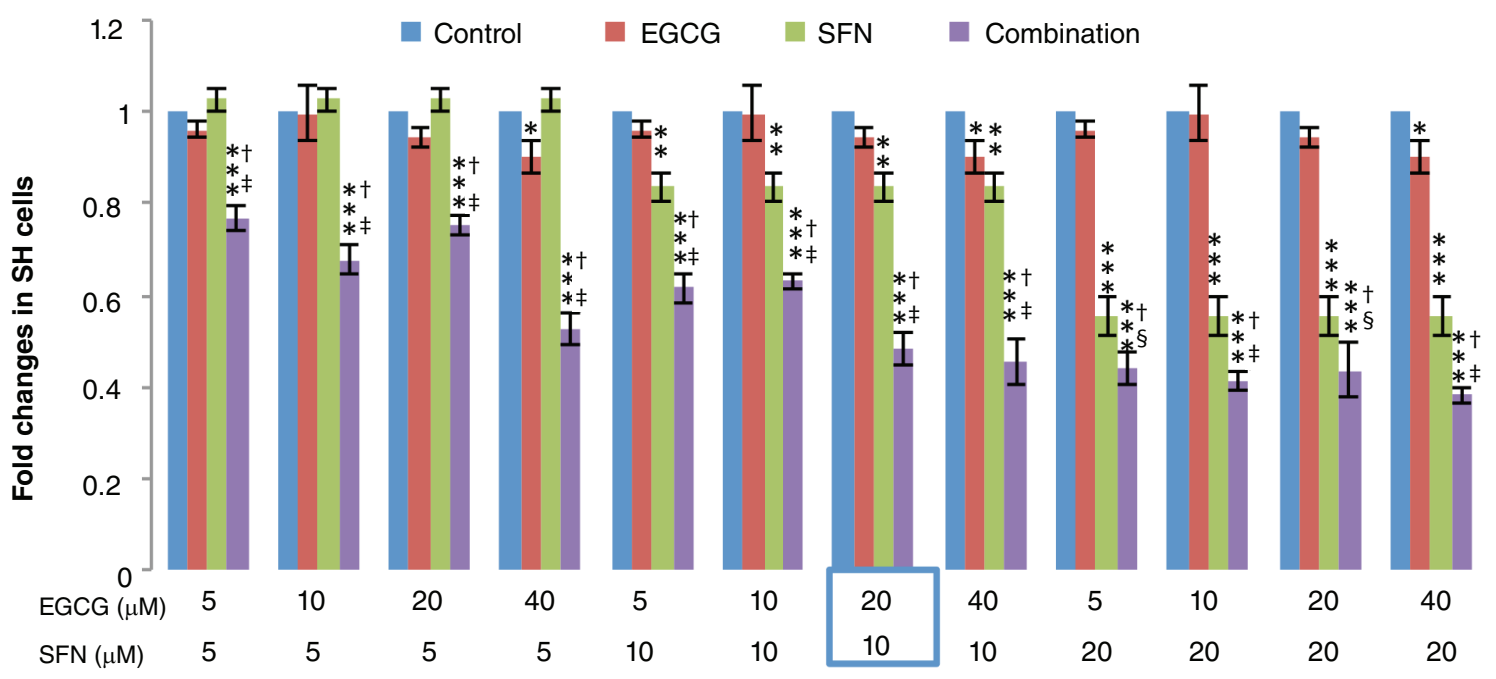

(B)

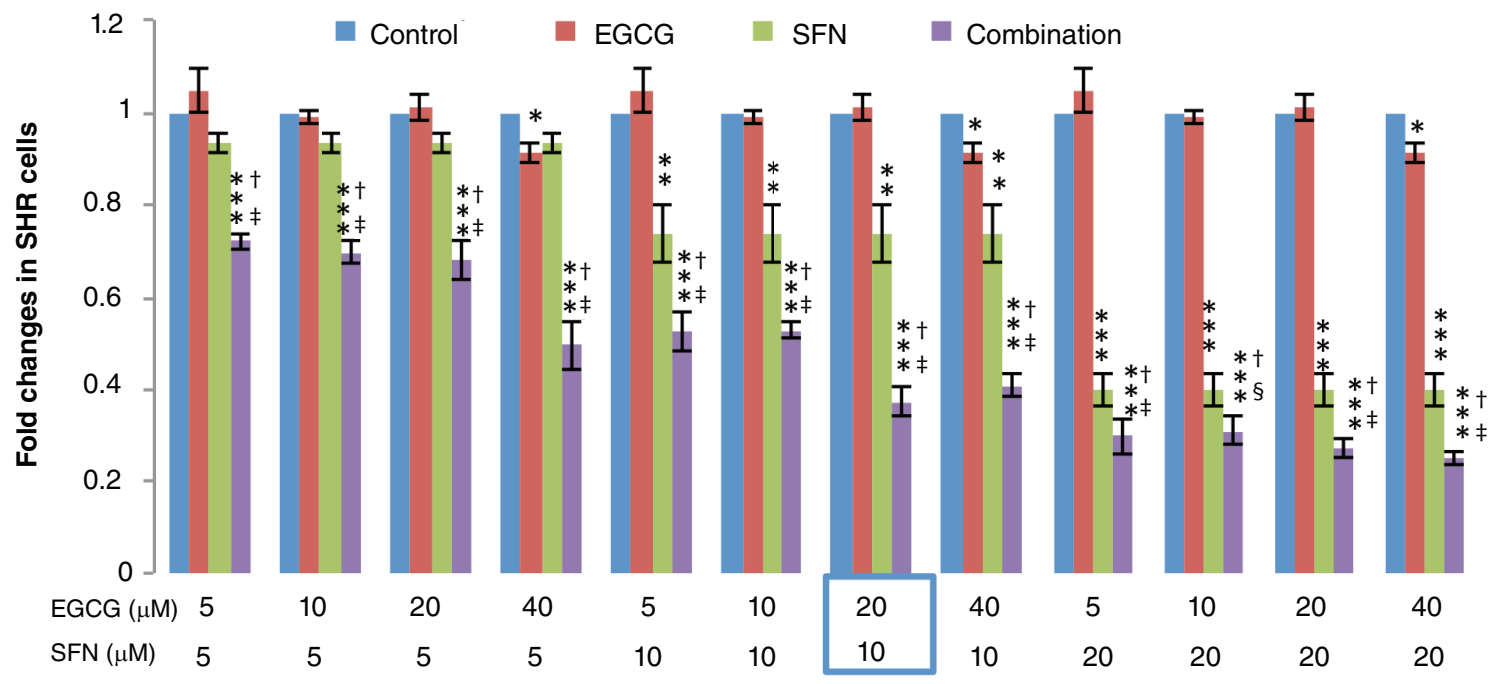

(C)

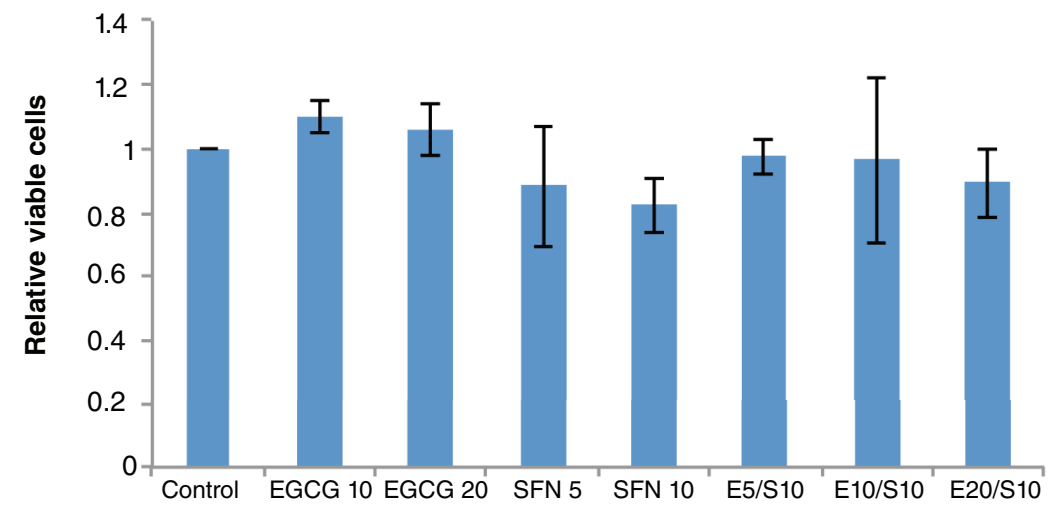


Figure 1. Growth inhibitory effects of EGCG and SFN combinatorial treatment (see facing page). SH (A) and SHR (B) cells were treated with different concentrations of EGCG and SFN, alone or in combination for 3 days. Normal human mammary epithelial cells (C) were used as a control to test for potential toxicity. The circled combination of EGCG and SFN represented the optimal concentrations of these two compounds with physiological availability as well as potential synergistic effects on breast cancer growth inhibition. The treatment concentrations of EGCG or SFN alone or in combination are illustrated at the bottom of each graph. Columns, mean; bars, standard deviation.

${ }^{\dagger} p<0.001$, significantly different from EGCG.

${ }^{\ddagger} p<0.001$, significantly different from SFN.

${ }^{s} \mathrm{p}<0.05$, significantly different from SFN.

${ }^{*} \mathrm{p}<0.05 ; * * \mathrm{p}<0.01 ; * * * \mathrm{p}<0.001$, significantly different from control.

EGCG: Epigallocatechin-3-gallate; SFN: Sulforaphane; SH cells: Breast precancerous cells, derived from normal HMECs transfected with SV4O and hTERT; SHR cells: Early transformed breast cancer cells, derived from normal HMECs transfected with SV4O, hTERT and H-Ras.

USA) were administered in drinking water; BSp diet: mice were fed with $26 \%$ of BSp diet (modified AIN93G diet supplemented with 26\% BSp seeds; TestDiet, MO, USA); combination diet: mice were fed with both GTPs and BSp diets. Mice were administered the corresponding experimental diets from 2 weeks prior to injection throughout the study. A $100 \mu \mathrm{l}$ of exponentially growing breast cancer SHR cells $\left(2 \times 10^{6}\right)$ was injected orthotopically into the second or third pair of the right thoracic mammary fat pad of each mouse to determine the in vivo efficacy of these diets on human breast tumor development.

Tumor sizes and body weight were measured weekly. Tumor volumes were calculated using the formula: tumor volume $\left(\mathrm{cm}^{3}\right)=\left(\right.$ length $\times$ width $\left.{ }^{2}\right) \times 0.523$ [23] . Tumor volume on day 1 (injection day) is considered as 1 . The experiment was terminated when the mean of tumor diameter in the control mice exceeded $1.0 \mathrm{~cm}$ following the guidelines of Institutional Animal Care and Use Committee at UAB. At the end of the experiment, the primary breast tumors were excised, weighed and appropriately stored for further analysis. Animal procedures were reviewed and approved by UAB Institutional Animal Care and Use Committee (Animal Project Number: 110109327).

\section{Immunohistochemical determination of tumor cell proliferation}

Primary breast tumor tissues were dissected and fixed in $10 \%$ buffer-neutralized formalin for histology and immunohistochemistry. Tumor slices $(5 \mu \mathrm{m}$ thick $)$ were deparaffinized and rehydrated in a series of graded alcohols. Antigens were retrieved in boiling $10 \mathrm{mmol} / \mathrm{l}$ sodium citrate buffer ( $\mathrm{pH}$ 6.0) for $20 \mathrm{~min}$. The slides were then washed in PBS and blocked with $1 \%$ bovine serum albumin. After blocking, the slides were incubated with antiproliferating cell nuclear antigen (PCNA; Cell Signaling Technology) for $2 \mathrm{~h}$ at room temperature. After washing in PBS, the sections were incubated with biotinylated secondary antibody for $45 \mathrm{~min}$ followed by horseradish peroxidase-conjugated streptavidin, incubated with diaminobenzidine substrate and counterstained with hematoxylin. Representative photographs were taken and the numbers of PCNA-positive cells were detected and counted by a licensed pathologist. Microscopic immunohistochemical analysis of breast tissue sections was performed using an Olympus BX41 microscope fitted with a Q-color 5 Olympus camera.

\section{Statistical analyses}

Statistical significance between treatment and control groups was evaluated by one-way ANOVA followed by Tukey's test for multiple comparisons by using GraphPad Prism 5.00 version. Values were presented as mean $\pm \mathrm{SD}$ and $\mathrm{p}<0.05$ was considered significant. Westernblot protein density was analyzed by Image J software developed by NIH.

\section{Results}

\section{EGCG \& SFN in combination significantly} inhibited initiation of breast tumorigenesis

To elucidate the combinatorial efficacy of the GTP EGCG and BSp SFN on cellular viability, we used a well-established cellular cancer transformation system that closely mimics the process of early human breast tumorigenesis. In this model, three defined genetic elements including SV4O, hTERT and H-Ras were introduced into normal HMECs to undergo a neoplastic transformation $[26,27]$. During this process, different stages of human mammary cancer cells were generated to represent cellular malignant progression during early breast carcinogenesis including breast precancerous cells (SH cells, transfected with SV4O and hTERT) and early transformed breast cancer cells (SHR cells, transfected with all three genetic elements) [23]. This system has been verified and successfully used in breast cancer research as a useful prevention as well as therapeutic in vitro model system [22-24]. Precancerous SH cells and early transformed breast cancer SHR cells were established in our laboratory at around 20 population doublings when experiments were initiated as reported previously [23].

We initiated our study to determine the optimal dose of combined EGCG and SFN treatment that will induce synergistic cellular growth inhibition in precancerous SH and early transformed breast cancer SHR 
cells without causing toxicity to normal breast epithelial cells. The MTT assay was performed with $\mathrm{SH}$ and SHR cells treated with various concentrations of EGCG and SFN as shown in Figure 1A \& B. The maximal treatment concentrations are $40 \mu \mathrm{M}$ for EGCG and $20 \mu \mathrm{M}$ for SFN based on practicable consumable doses of these diets and our previous findings in other breast cancer cell lines [14,15]. We found that SFN treatment alone significantly inhibited cellular growth in precancerous $\mathrm{SH}$ cells and early transformed breast cancer SHR cells in a dose-dependent manner, whereas EGCG treatment alone can only affect cellular growth at the highest treatment concentration both in $\mathrm{SH}$ and SHR cells (Figure 1A \& B). Strikingly, all combination treatments induced significant cellular growth inhibition compared with control, EGCG and SFN alone, suggesting that combinatorial treatment with GTPs and BSp are more efficacious in suppressing breast cancer initiation than any of these two compounds acting alone and that a potential synergistic inhibitory effect is induced. We finally chose optimal combinatorial treatment concentrations of $20 \mu \mathrm{M}$ EGCG and $10 \mu \mathrm{M}$ SFN in both precancerous SH and early transformed breast cancer SHR for our subsequent studies due to the relatively low concentrations for each treatment and the more effective tumor inhibitory rate by this combinatorial regimen. Furthermore, this optimal combinatorial treatment concentration exhibits a synergistic effect on inhibiting cell proliferation in both precancerous $\mathrm{SH}$ (Combination Index $[\mathrm{CI}]=0.56$ ) and early transformed breast cancer SHR $(\mathrm{CI}=0.53)$ as determined by an online software, CompuSyn software, in which $\mathrm{CI}<1,=1$ and $>1$ indicate synergism, additive effect and antagonism, respectively [28].

We also evaluated the potential toxicity of this combination in normal breast HMECs (Figure 1C) and found that the tested concentrations of EGCG and SFN did not cause inhibitory effects on cell viability in HMECs indicating this treatment is likely safe. This dietary combination regimen using relatively low concentrations of EGCG and SFN is physiologically accessible and practical for humans as it represents only 74-148 $\mathrm{ml} /$ dose of $0.5 \%$ GTPs or $12-23 \mathrm{~g} \mathrm{BSp} /$ serving consumed per day by an adult human [29,30]. Based on these results, we therefore chose to use $20 \mu \mathrm{M}$ EGCG and $10 \mu \mathrm{M}$ SFN as optimal combined concentrations in our subsequent studies.

Combined treatment with EGCG \& SFN induced cellular apoptosis in early transformed breast cancer SHR cells

To address how combined treatment with EGCG and SFN affects cellular proliferation, we conducted cell apoptosis assays on precancerous $\mathrm{SH}$ cells and early transformed breast cancer SHR cells with the aforementioned treatments. As indicated in Figure 2A \& B (right panel), we found that either EGCG or SFN treatment alone or in combination resulted in significant apoptosis in breast cancer SHR cells $(\mathrm{p}<0.05)$, but combination treatment induced a more prominent apoptosis compared with control cells $(\mathrm{p}<0.01)$ with a synergistic effect $(\mathrm{CI}=0.05)$. However, these treatments did not induce significant cellular apoptosis in precancerous SH cells as shown in Figure 2A \& B (left panel). In addition, the optimal combined treatment did not cause a dramatic increase in apoptosis rate $(<20 \%)$ indicating less toxicity and more safe practical potential. Consistent with the results of cell viability shown in Figure 1, we speculated that this dietary regimen may exhibit greater efficacy in inducing cellular growth inhibition and apoptosis in early transformed SHR cells than pre-transformed SH cells, indicating its potent chemotherapeutic and chemopreventive potential against breast tumorigenic cells.

\section{Combinatorial treatment with EGCG \& SFN induced cell cycle arrest}

To further investigate the effects of this dietary combination regimen on the cell cycle, the cell cycle profiles of precancerous $\mathrm{SH}$ cells and early transformed breast cancer SHR cells were analyzed using flow cytometry. We found EGCG and SFN treatment resulted in a significant increase in the percentage of cells in the $S$-phase in precancerous $\mathrm{SH}$ cells $(\mathrm{p}<0.001$; Figure $3 \mathrm{~A} \& \mathrm{C})$, whereas it led to significantly cell accumulation in both S- $(\mathrm{p}<0.001)$ and G2/M-phase $(\mathrm{p}<0.01)$ in breast cancer SHR cells (Figure 3B \& D). Compared with EGCG treatment alone, SFN treatment alone is more effective in inducing cell cycle arrest indicating SFN may play a dominating role on suppressing breast cancer development in this combination regimen. These results suggest that combinatorial dietary regimen of EGCG and SFN mediates cell growth inhibition by inducing cell cycle arrest, especially in completed transformed breast cancer cells.

\section{Epigenetic enzymatic expression \& activity} changes in response to EGCG \& SFN treatments To further interpret the mechanisms of epigenetic modulations by EGCG and/or SFN treatment during breast tumorigenesis, we assessed gene expression and enzymatic activities of two important epigeneticassociated enzymes involved in regulation of histone acetylation and DNA methylation such as HDACs and DNMTs, respectively. As shown in Figure 4A-D, right panels, EGCG and SFN treatment significantly reduced gene expressions and enzymatic activities of both HDAC1 and DNMT1 in early transformed 
(A)
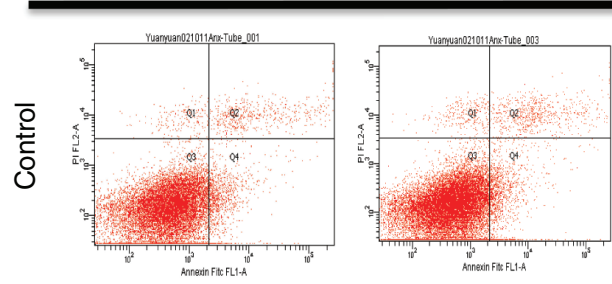

导
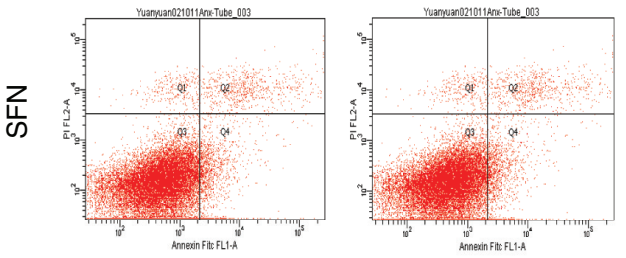

(B)

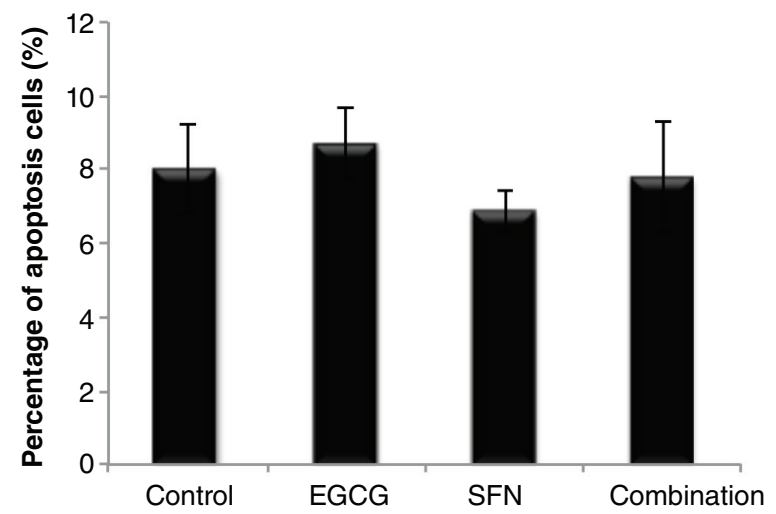

$\mathrm{SH}$
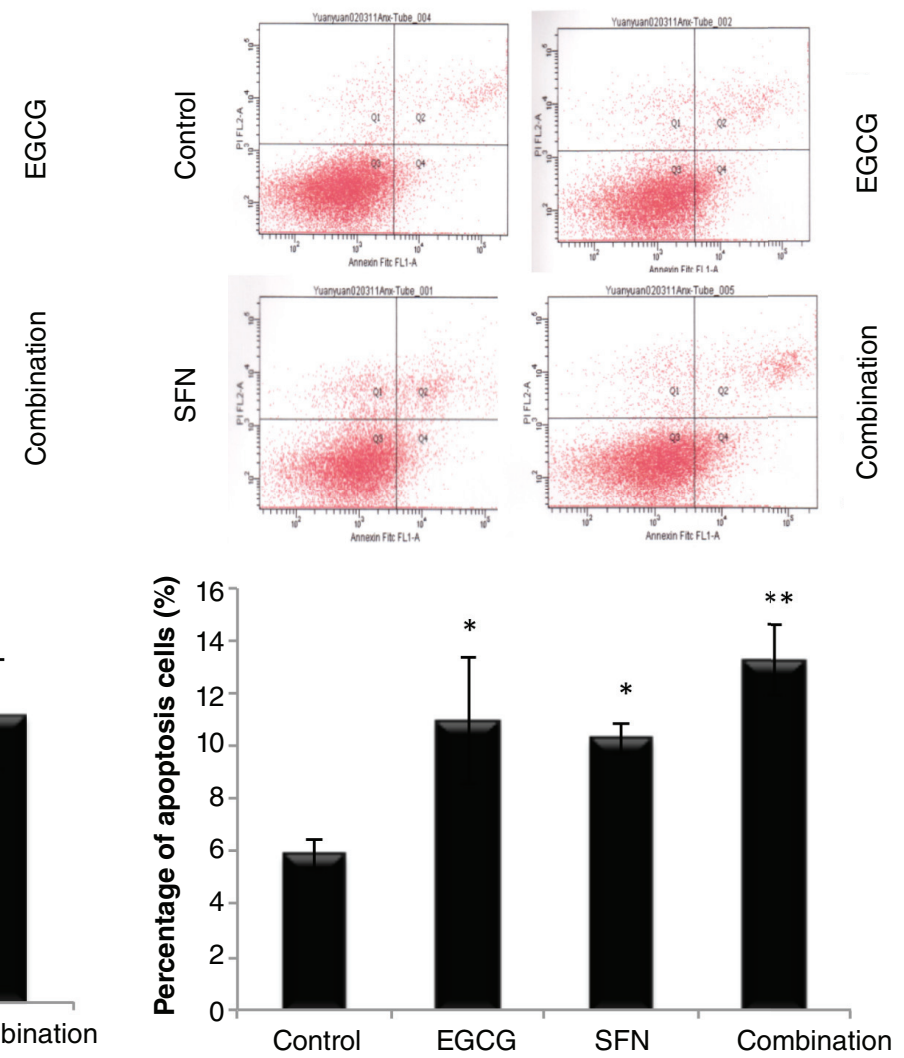

SHR
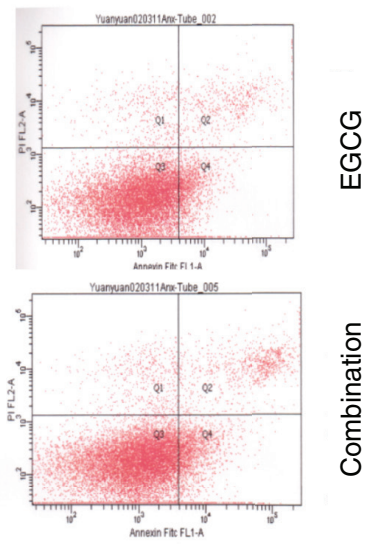

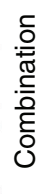

Figure 2. Apoptosis analysis by combined treatment with EGCG and SFN. The concentrations of the combination treatment were $20 \mu \mathrm{M}$ EGCG and $10 \mu \mathrm{M}$ SFN based on synergistic effects of breast cancer growth inhibition as shown in Figure 1. Cellular apoptosis was detected by using an annexinV and PI staining system. (A) Becton Dickinson FACSCalibur Flow Cytometer was used to acquire and analyze a minimum of 105 events using the CellQuest program. The graphs are representative of three independent experiments. (B) Histogram of the apoptosis rate in SH (left panel) and SHR (right panel) cells in response to treatments with EGCG and SFN alone and combination. Columns, mean; bars, standard deviation.

$* p<0.05 ; * * p<0.01$ versus control.

EGCG: Epigallocatechin-3-gallate; SFN: Sulforaphane; SH cells: Breast precancerous cells, derived from normal HMECs transfected with SV4O and hTERT; SHR cells: Early transformed breast cancer cells, derived from normal HMECs transfected with SV4O, hTERT and H-Ras.

SHR cells, suggesting that this dietary combination treatment may exert its antibreast cancer properties through, at least in part, regulation of epigenetic pathways. The combinatorial treatment was more effective in inhibiting gene expressions and enzymatic activities of HDAC1 and DNMT1 compared with control, EGCG and SFN treatment alone. In addition, we found EGCG and SFN in combination can only induce significant changes of these epigenetic modulators in early transformed breast cancer SHR cells (Figure 4A-D, right panels) but not in precancerous
SH cells (Figure 4A-D, left panels), which is consistent with our abovementioned findings that this dietary regimen is more effective on early transformed breast cancer cells. Unlike the transformed breast cancer SHR cells, the expressions of HDAC1 and DNMT1 in precancerous $\mathrm{SH}$ cells are not matched by the relevant enzymatic activities suggesting a different epigenetic regulatory mechanism may be involved in these two cells during early breast cancer initiation.

Although there is no synergistic effect found in the activities of DNMT1 and HDAC1 in transformed 


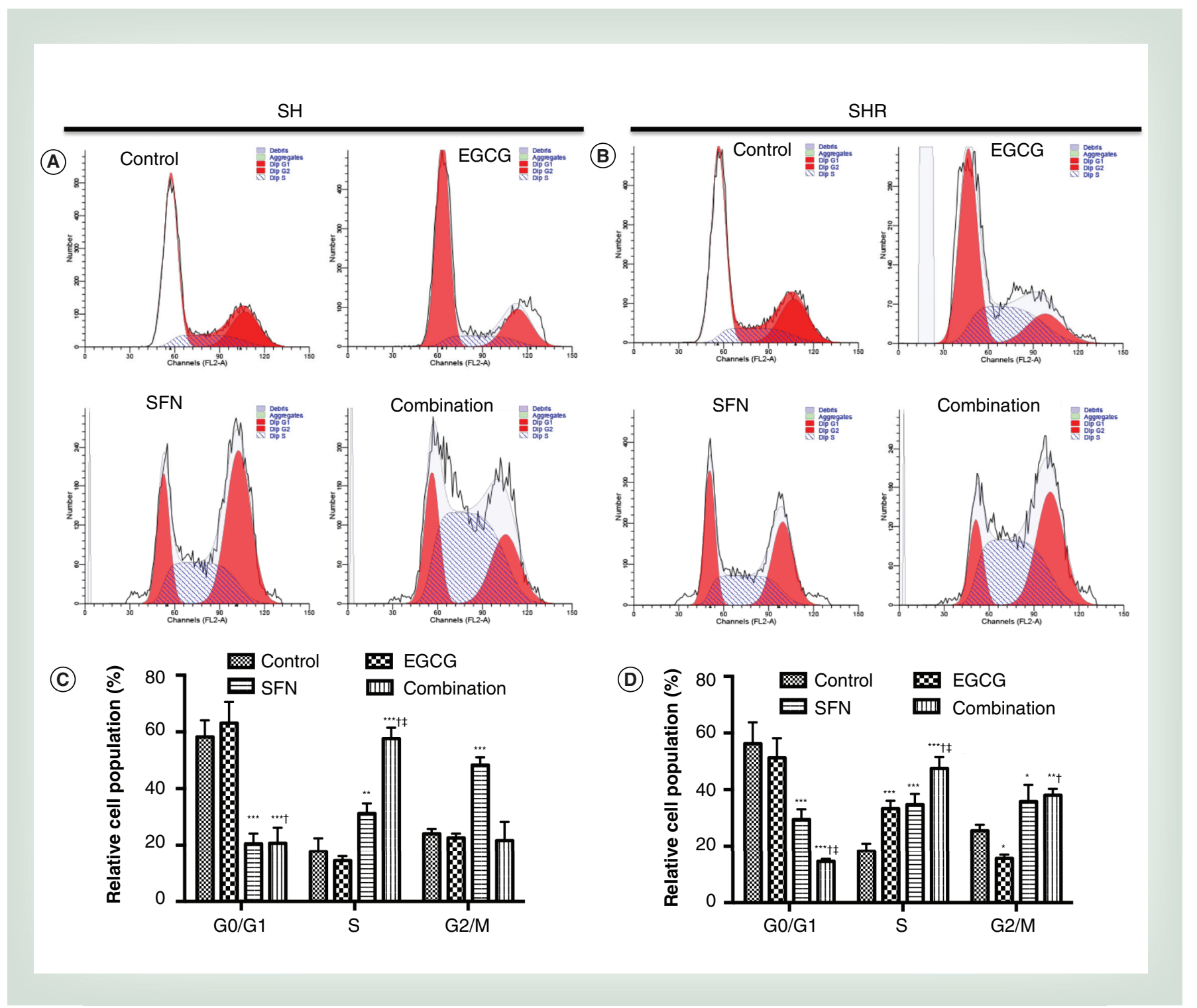

Figure 3. Effects of combinatorial treatment with EGCG and SFN on cell cycle distribution. Precancerous SH cells (A) or early transformed breast cancer SHR cells (B) were treated with EGCG $(20 \mu \mathrm{m})$ and SFN $(10 \mu \mathrm{m})$, alone or in combination for 3 days followed by propidium iodide-based flow cytometric analysis. Bar charts reveal the percentage of cells in the indicated phases of the cell cycle in SH cells (C) and SHR cells (D). The representative graphs are obtained from three independent experiments. Columns, mean; bars, standard deviation. ${ }^{\dagger} p<0.01$, significantly different from EGCG.

${ }^{\ddagger} \mathrm{p}<0.01$, significantly different from SFN.

${ }^{*} \mathrm{p}<0.05 ; * * \mathrm{p}<0.01 ; * * * \mathrm{p}<0.001$, significantly different from control.

EGCG: Epigallocatechin-3-gallate; SFN: Sulforaphane; SH cells: Breast precancerous cells, derived from normal HMECs transfected with SV4O and hTERT; SHR cells: Early transformed breast cancer cells, derived from normal HMECs transfected with SV4O, hTERT and H-Ras.

breast cancer SHR cells (Figure 4C \& D, right panels), we found an additive effect $(\mathrm{CI}=1)$ in expression of $H D A C 1$ (Figure 4A, right panel) and a synergistic effect $(\mathrm{CI}=0.67)$ in DNMT1 expression (Figure $4 \mathrm{~B}$, right panel) in response to combined treatment with EGCG and SFN. Based on our previous studies, EGCG is a strong inhibitor for DNMTs but has relative weak inhibitory effects on HDACs $[14,20]$, whereas SFN has shown a strong inhibitory effect on HDACs [15,21].
In Figure 4A \& C, right panels, both EGCG and SFN treatment alone significantly reduced HDAC1 expressions and enzymatic activities in SHR cells because both of these compounds are HDACs inhibitors, and combination leads to a further reduction. However, SFN treatment alone cannot induce significant changes in DNMT1 expression and enzymatic activity (Figure 4B \& D, right panels) due to its limited impact on DNMTs. This inhibitory effect is strengthened 
when SFN is combined with EGCG indicating an interaction between these two compounds may act in regulation of epigenetic pathways. These results indicate that EGCG and SFN treatment may affect epi- genetic pathways most likely via influencing histone acetylation and DNA methylation leading to a reversal of aberrant epigenetic profiles during early breast tumorigenesis and cancer suppression.
(A)

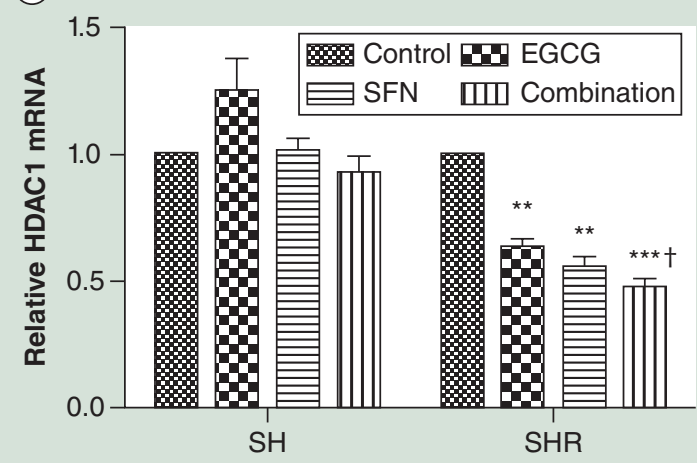

(C)

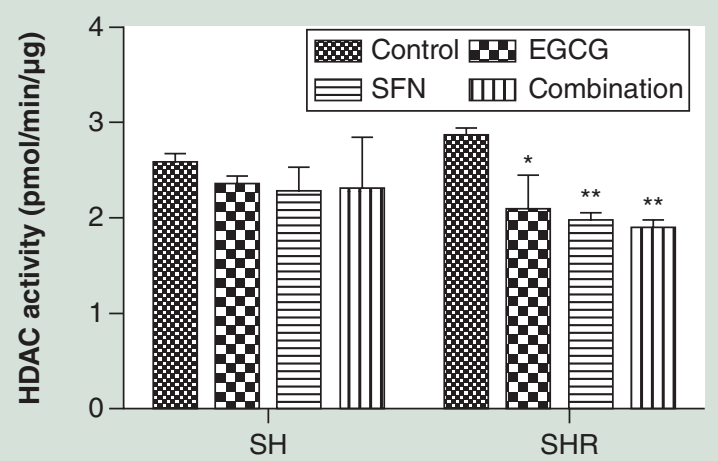

(E)

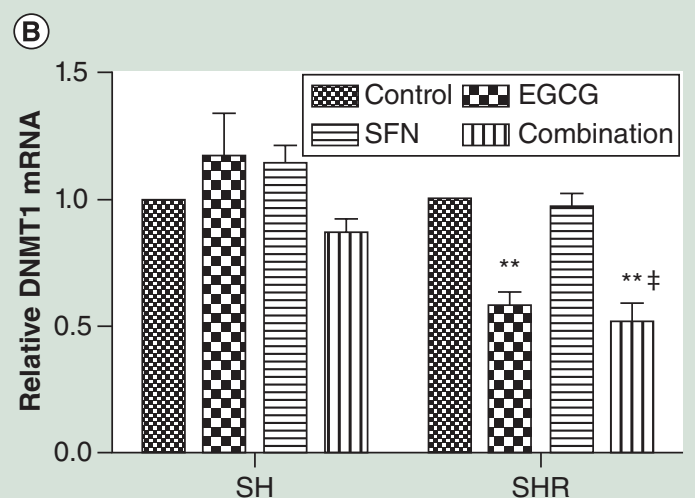

(D)

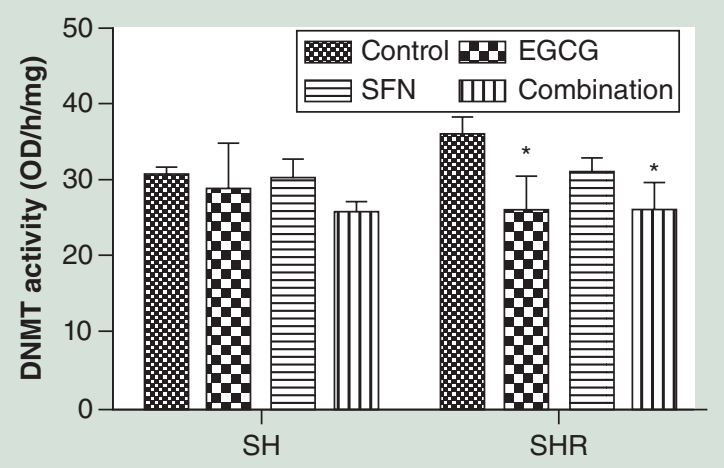

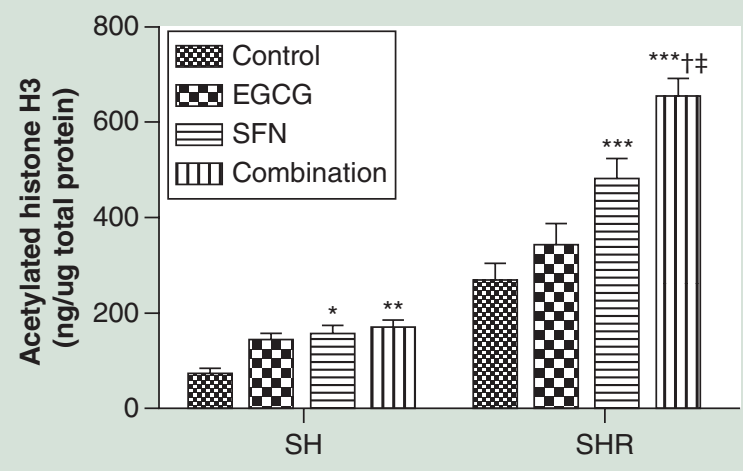

Figure 4. Relative mRNA expression and enzymatic activities of HDAC1 and DNMT1 as well as global histone H3 acetylation level by EGCG and/or SFN treatment. Precancerous SH cells and early transformed breast cancer SHR cells were treated with EGCG $(20 \mu \mathrm{m})$ and SFN $(10 \mu \mathrm{m})$, alone or combination. Quantitative real-time PCR was performed to measure relative transcription of HDAC1 (A) and DNMT1 (B). Data were in triplicate from three independent experiments and were normalized to GAPDH and calibrated to untreated samples. (C) HDACs enzymatic activity; (D) DNMTs enzymatic activity. (E) Global histone H3 acetylation level. The values of enzymatic activities or acetylated histone $\mathrm{H} 3$ were derived from the means of three independent experiments. Columns, mean; bars, standard deviation.

${ }^{+} p<0.01$, significantly different from EGCG.

${ }^{\ddagger} p<0.01$, significantly different from SFN.

${ }^{*} p<0.05 ; * * p<0.01 ; * * * p<0.001$, significantly different from control.

DNMT1: DNA methyltransferase 1; EGCG: Epigallocatechin-3-gallate; HDAC1: Histone deacetylases 1;

SFN: Sulforaphane; SH cells: Breast precancerous cells, derived from normal HMECs transfected with SV4O and $h T E R T$; SHR cells: Early transformed breast cancer cells, derived from normal HMECs transfected with SV40, hTERT and H-Ras. 
EGCG \& SFN in combination increased global histone $\mathrm{H} 3$ acetylation level

Although we observed significant changes in epigenetic modulators by dietary EGCG and SFN treatments (Figure 4A-D), we next sought to investigate further epigenetic mechanisms regulated by this combinatorial treatment during breast tumorigenesis. We evaluated global histone acetylation level by measuring global acetylation of histone $\mathrm{H} 3$, which is the most common acetyl-modified histone. As shown in Figure 4E, combined treatment with EGCG and SFN significantly increased global acetylation of histone $\mathrm{H} 3$ in both precancerous $\mathrm{SH}$ cells and early transformed breast cancer SHR cells, and this increment is more obvious in SHR cells $(p<0.001$, significantly different from control, EGCG and SFN) than in SH cell ( $p<0.01$, significantly different from control), which is consistent with our previous results that this dietary regimen is more effective on breast cancer cells. This result suggests that our novel dietary combination regimen results in an open chromatin structure leading to an alteration of global gene expression profile that may contribute to its antibreast cancer properties through, at least in part, repression of HDAC1 expression and/or activity.

\section{Genome-wide DNA methylation alterations in response to EGCG \& SFN treatment}

We further extended our study by testing genome-wide DNA methylation alterations in early transformed breast cancer SHR cells in response to EGCG and/ or SFN treatment. To investigate methylation status between different treatment groups, hierarchical clustering was conducted on 266 selected probes for all single and combined treatments with absolute $\beta$ difference greater than 0.2 compared with the control treatment (Figure 5A). Among them, 51 probes in combination are hypermethylated and 44 are hypomethylated. The clusters showed significant variation of DNA methylation status between control, EGCG, SFN and combination groups suggesting epigenetic mechanisms may play a role in dietary EGCG and SFN-associated breast cancer preventive and therapeutic effects. Functional gene ontology analysis (Figure 5C) indicates multiple cellular pathways have been modulated, such as chromosomal structure, RNA binding and differentiation, that may eventually contribute to breast cancer prevention effects by this combinatorial dietary treatment. To identify individual gene loci whose methylation status were correlated with EGCG and SFN treatment-induced breast cancer inhibition, we selected 21 genes with significant $\beta$ changes in response to EGCG and SFN treatment which also have been documented to be associated with cancer development and controlled by epigenetic factors (Figure 5B) [31-54]. As illustrated in Table 1, we observed that EGCG and SFN treatment induced DNA methylation alterations on several genes that may correlate with this dietary regimen-induced breast carcinogenesis suppression. In addition, we discovered a greater change of DNA methylation status in these tumor-related genes by the combination treatment with EGCG and SFN than any of these two compounds acting alone. Collectively, these results suggest that combinatorial treatment with EGCG and SFN can affect epigenetic markers of key tumor-related genes leading to inhibition of breast tumor initiation and development.

\section{Combinatorial treatment with EGCG \& SFN} caused expression changes in tumor-related genes due to regulation of epigenetic pathways

To further verify the correlation of DNA methylation status with actual gene expression, we evaluated expression of selected tumor-related genes in which the DNA methylation status are significantly altered by the treatment of EGCG and SFN as shown in Figure 5 \& Table 1. Two important tumor-related genes were chosen to represent tumor suppressor gene such as DCBLD2 [51] and tumor promoting gene such as SPET9 (Septin 9) [38,39]. We found that EGCG and SFN treatment alone and in combination significantly upregulated tumor suppressor gene DCBLD2 (Figure 6A) but downregulated tumor promoting gene Septin 9 (Figure 6B) in early transformed breast cancer SHR cells. These gene expression changes are positively associated with their DNA methylation status alterations suggesting epigenetic mechanisms may be involved in dietary EGCG and SFN-induced breast cancer suppression. We extended our experiment to further illustrate potential epigenetic mechanisms linking this novel combination treatment to its inhibitory effects on early breast cancer initiation. We found that combinatorial treatment-induced protein expression in DCBLD2 and Septin 9 was consistent with mRNA expression as shown Figure 6A \& B. DNMT1 and HDAC1 knockout studies showed upregulation of DCBLD2 and Septin 9 protein expression (Figure 6C-F). However, combinatorial treatment with EGCG and SFN significantly reinforced protein increment of the tumor suppressor gene, DCBLD2, when two epigenetic modulators, DNMT1 (Figure 6C \& E) and HDAC1 (Figure 6D \& F), are suppressed. On the contrary, EGCG and SFN treatment attenuates the activation effects of repressed DNMT1 and HDAC1 on protein expression of the tumor promoting gene, Septin 9. These results further indicate that combined GTPs/EGCG and BSp/SFN are highly effective in inhibiting early breast cancer development by, at least in part, regulating epigenetic mechanisms. 
(A)

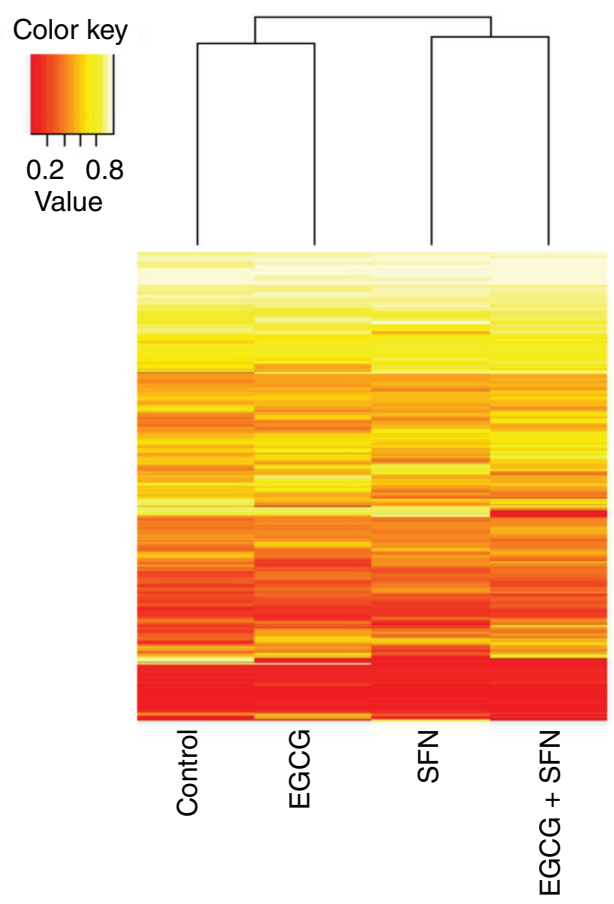

(B)

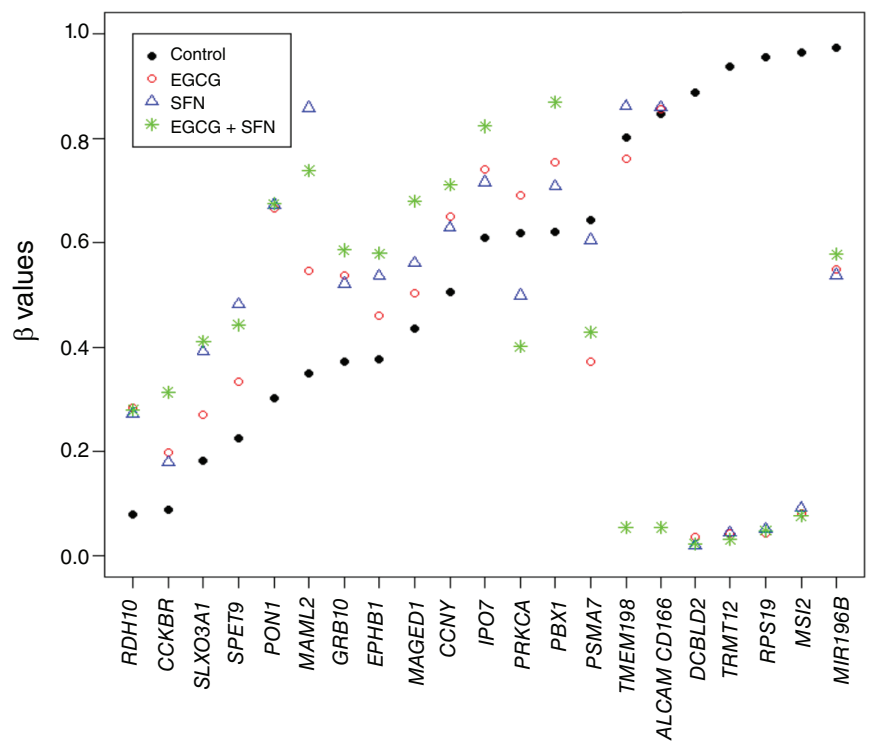

(C)

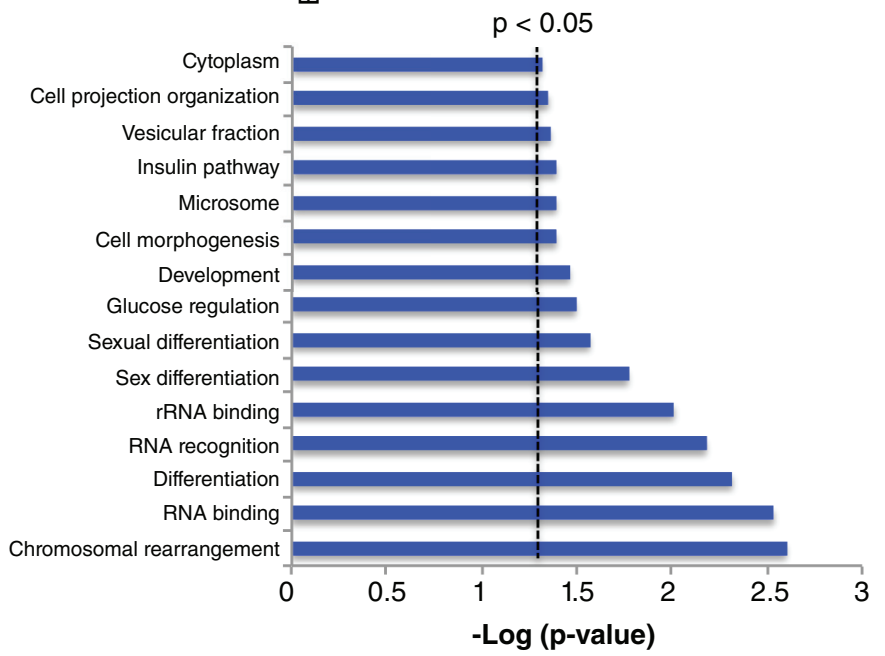

Figure 5. Differential DNA methylation status in early transformed breast cancer SHR cells. (A) Hierarchical clustering using BeadChip data cross four treatment groups (control, EGCG, SFN and combination) in SHR cells. A total of 266 selected probes were presented with absolute $\beta$ difference greater than 0.2 compared with the control treatment. Each row represents a probe; each column represents a treatment. The level of DNA methylation ( $\beta$ value) is represented with a color scale as depicted above the heatmap.

(B) Scatter plot illustrating distribution of methylation for selected genes (Table 1 ) with significant $\beta$ changes in response to EGCG and SFN treatment. $\beta$ values for different treatment groups are represented as the symbols with different colors and shapes as depicted in the legend. (C) Gene functional associations by gene ontology analysis by DAVID. Dotted line represents a threshold for $p<0.05$. EGCG: Epigallocatechin-3-gallate; SFN: Sulforaphane.

\section{Dietary GTPs \& BSp inhibited the growth of breast cancer xenografts}

To determine the in vivo breast cancer inhibitory properties of dietary GTPs and BSp, we conducted animal experiments to examine whether dietary administration of either GTPs and BSp alone or in combination can inhibit the growth of breast cancer xenografts. We used early transformed breast cancer cells, SHR, to 
Table 1. Selected tumor- or epigenetic-related genes with significant $\beta$ changes in response to epigallocatechin-3gallate and sulforaphane treatment.

\begin{tabular}{|c|c|c|c|c|}
\hline $\begin{array}{l}\text { UCSC ref gene } \\
\text { name }\end{array}$ & Gene full name & $\begin{array}{l}\text { Methylation status } \\
\text { change }\end{array}$ & Gene function & Ref. \\
\hline${ }^{\Omega} M A M L 2$ & Mastermind-like 2 & Hypermethylation & Promote tumor growth & [31] \\
\hline ^PON1 & Paraoxonase 1 & Hypermethylation & $\begin{array}{l}\text { Multifactorial antioxidant enzyme paraoxonase } \\
\text { family; SNP variation is related to breast cancer; } \\
\text { methylation regulation is involved }\end{array}$ & [32] \\
\hline$\Omega P B X 1$ & $\begin{array}{l}\text { Pre-B-cell leukemia } \\
\text { homeobox } 1\end{array}$ & Hypermethylation & $\begin{array}{l}\text { Relevant to ER-positive breast cancer progression; } \\
\text { regulated by epigenetic factors }\end{array}$ & {$[33,34]$} \\
\hline${ }^{\xi} M A G E D 1$ & $\begin{array}{l}\text { Melanoma antigen } \\
\text { family } D, 1\end{array}$ & Hypermethylation & $\begin{array}{l}\text { Inhibit tumor growth; regulated by epigenetic } \\
\text { mechanisms }\end{array}$ & [35] \\
\hline eSLCO3A1 & $\begin{array}{l}\text { Solute carrier organic } \\
\text { anion transporter family, } \\
\text { member } 3 A 1\end{array}$ & Hypermethylation & $\begin{array}{l}\text { Breast cancer regulated gene; regulated by } \\
\text { epigenetic mechanisms }\end{array}$ & [36] \\
\hline${ }^{\xi} \Omega C C K B R$ & Cholecystokinin B receptor & Hypermethylation & $\begin{array}{l}\text { Promote breast cancer; regulated by epigenetic } \\
\text { factors }\end{array}$ & [37] \\
\hline${ }^{\lambda S E P T 9}$ & Septin 9 & Hypermethylation & $\begin{array}{l}\text { Tumor-related gene, regulated by epigenetic } \\
\text { mechanisms }\end{array}$ & {$[38,39]$} \\
\hline "GRB10 & $\begin{array}{l}\text { Growth factor } \\
\text { receptor-bound protein } 10\end{array}$ & Hypermethylation & $\begin{array}{l}\text { Tumor-related gene, regulated by epigenetic } \\
\text { mechanisms }\end{array}$ & [40] \\
\hline$\delta / P O 7$ & Importin 7 & Hypermethylation & $\begin{array}{l}\text { Tumor-related gene, regulated by epigenetic } \\
\text { mechanisms }\end{array}$ & {$[41,42]$} \\
\hline${ }^{\lambda} \mathrm{CCNY}$ & Cyclin Y & Hypermethylation & Tumor promoting gene & {$[43]$} \\
\hline${ }^{\lambda} E P H B 1$ & EPH receptor $\mathrm{B} 1$ & Hypermethylation & $\begin{array}{l}\text { Loss of expression in gastric carcinoma associated } \\
\text { with invasion and metastasis }\end{array}$ & {$[44]$} \\
\hline "RDH10 & Retinol dehydrogenase 10 & Hypermethylation & $\begin{array}{l}\text { Tumor-related gene, regulated by epigenetic } \\
\text { mechanisms }\end{array}$ & {$[45]$} \\
\hline${ }^{*} P S M A 7$ & $\begin{array}{l}\text { Proteasome subunit, alpha } \\
\text { type, } 7\end{array}$ & Hypomethylation & Tumor suppressor gene & {$[46]$} \\
\hline IPRKCA & Protein kinase C, alpha & Hypomethylation & Breast tumor related gene & [47] \\
\hline "MIR196B & MicroRNA 196b & Hypomethylation & Oncogene & [48] \\
\hline *TMEM198 & $\begin{array}{l}\text { Transmembrane protein } \\
198\end{array}$ & Hypomethylation & Related with Wnt pathway & [49] \\
\hline${ }^{\Omega} A L C A M C D 166$ & $\begin{array}{l}\text { Activated leukocyte cell } \\
\text { adhesion molecule CD166 }\end{array}$ & Hypomethylation & Strong relevance to breast cancer & [50] \\
\hline "DCBLD2 & $\begin{array}{l}\text { Discoidin, CUB and LCCL } \\
\text { domain containing } 2\end{array}$ & Hypomethylation & $\begin{array}{l}\text { Tumor suppressor gene, regulated by epigenetic } \\
\text { mechanisms }\end{array}$ & [51] \\
\hline $\mathrm{e} M S / 2$ & $\begin{array}{l}\text { Musashi RNA-binding } \\
\text { protein } 2\end{array}$ & Hypomethylation & Tumor-related genes & [52] \\
\hline \#RPS19 & Ribosomal protein $\mathrm{S} 19$ & Hypomethylation & Tumor-related genes & [53] \\
\hline${ }^{2} T R M T 12$ & $\begin{array}{l}\text { Trna methyltransferase } 12 \\
\text { homolog }\end{array}$ & Hypomethylation & Overexpression in breast cancer & [54] \\
\hline
\end{tabular}

grow xenografts in athymic nude mice that had been fed $0.5 \%$ GTPs in drinking water and/or a diet supplemented with $26 \%$ BSp for two weeks before injection of the tumor cells and the treatment continued throughout the study. The concentrations of the diets used in this study are converted from our in vitro study. They are considered physiologically available and correspond to drinking 2-3 cups (2.5 $\mathrm{mg}$ EGCG/ml water) of green tea and consuming $266 \mathrm{~g}$ ( $\sim 4$ cups) $\mathrm{BSp} /$ per day by an adult human, respectively $[55,56]$. 


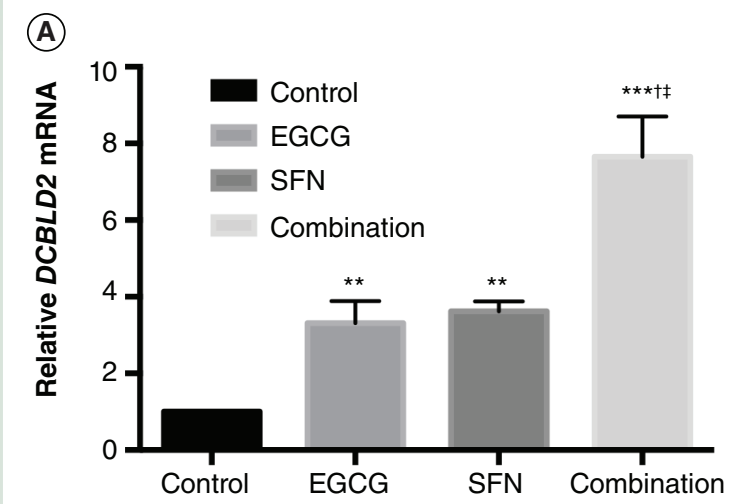

(C)

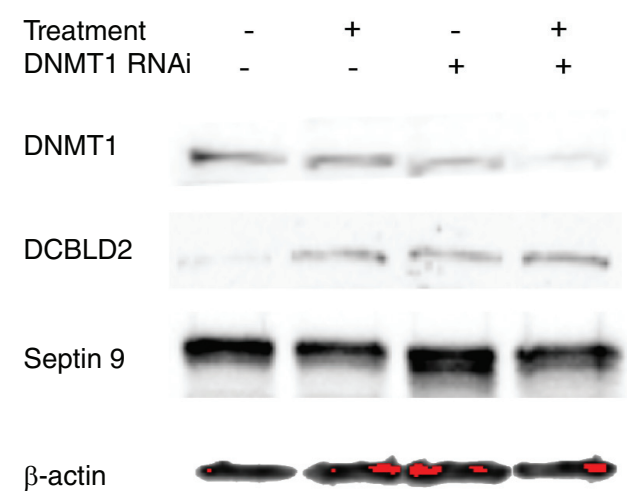

(E)

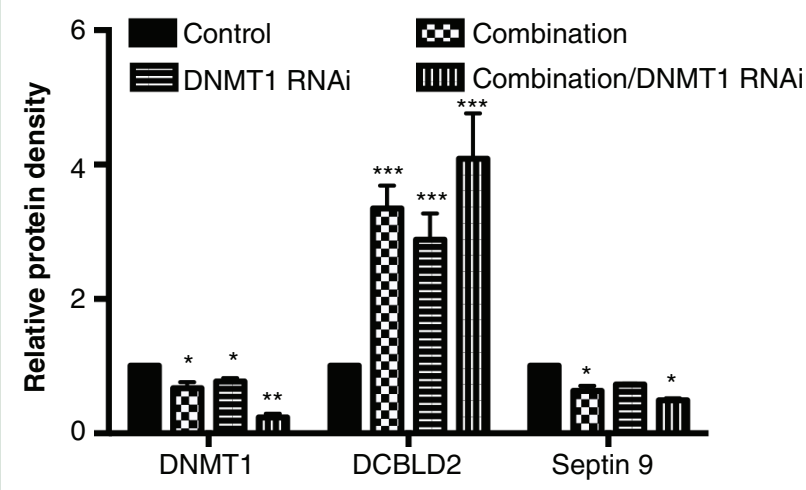

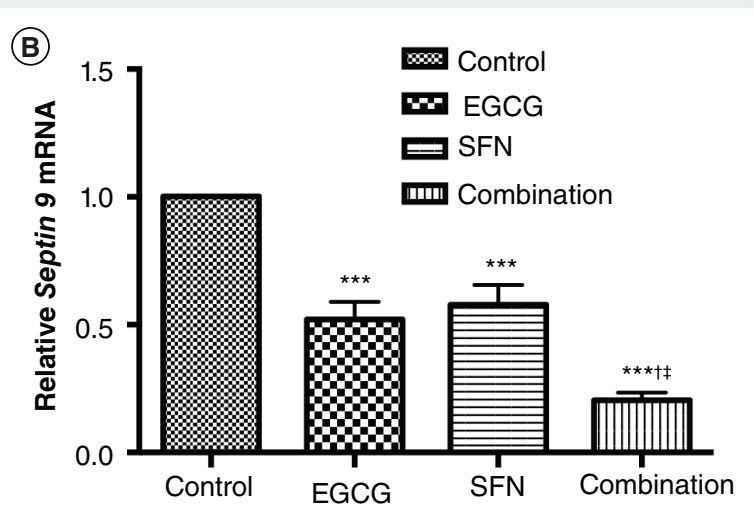

(D)

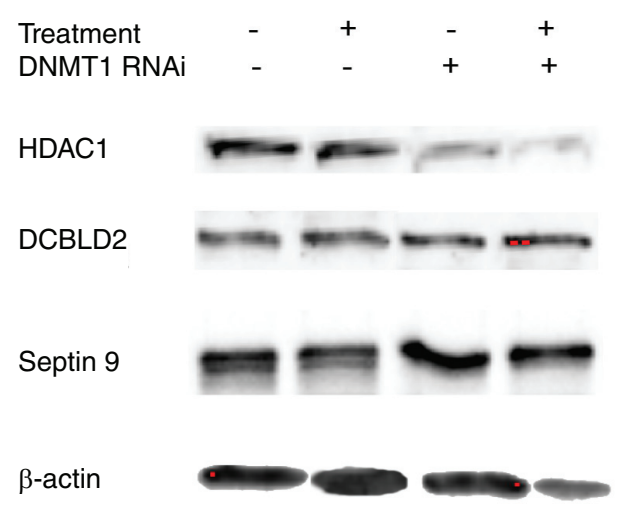

(F)

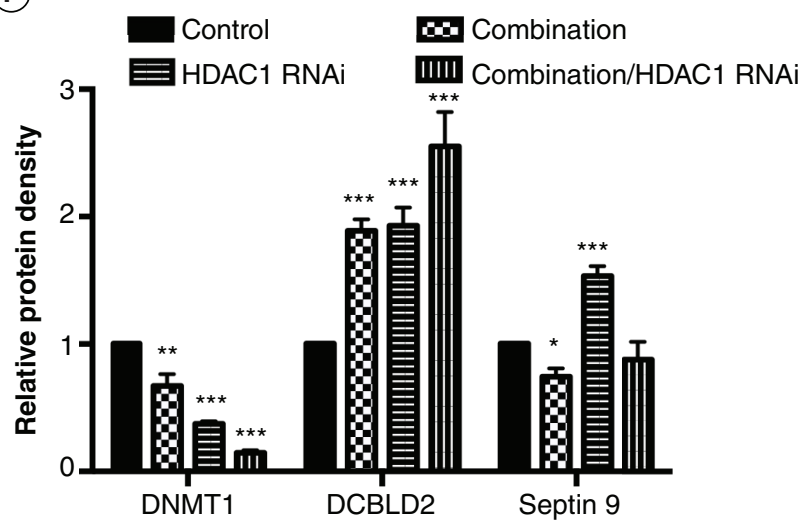

Figure 6. Combinatorial treatment with EGCG and SFN caused expression changes in tumor-related genes through regulation of HDAC1 and DNMT1. (A) and (B) quantitative real-time PCR was performed to measure relative transcription of DCBLD2 (A) and Septin 9 (B) in early transformed breast cancer SHR cells treated with EGCG $(20 \mu \mathrm{m})$ and SFN $(10 \mu \mathrm{m})$ alone or in combination. (C \& D) Protein expression of DCBLD2 and Septin 9 in response to suppressed DNMT1 (C) or HDAC1. (D) Combination-treated or untreated SHR cells were transfected with either DNMT1 siRNA (C) or HDAC1 siRNA (D) to inhibit related gene expression and extracted protein after 3 days of transfection. (E \& F) protein quantification for $C(E)$ and $D(F)$. Data were in triplicate from three independent experiments and normalized to internal control and calibrated to levels in untreated samples. Columns, mean; bars, standard deviation.

${ }^{\dagger} p<0.01$, significantly different from EGCG.

${ }^{\ddagger} p<0.01$, significantly different from SFN.

$* * p<0.01 ; * * * p<0.001$, significantly different from control.

DNMT1: DNA methyltransferase 1; EGCG: Epigallocatechin-3-gallate; HDAC1: Histone deacetylases 1; SFN: Sulforaphane. 
Periodic measurement of the tumor volume indicated that both dietary GTPs and BSp significantly suppressed tumor growth from as early as 3 week post injection. However, combinatorial treatment of GTPs and BSp was significantly more efficacious in inhibiting breast xenograft growth throughout the experimental time frame with an overall $93.87 \%$ inhibition rate compared with $74.89 \%$ for GTPs alone and $58.22 \%$ for BSp alone (Figure 7A \& Table 2). It also indicates that combined GTPs and BSp lead to a synergistic effect on tumor inhibition $(\mathrm{CI}=0.167)$. The wet weight of the SHR xenograft tumors per mouse was significantly lower in the mice administered either GTPs or BSp diet, especially GTPs and BSp in combination, as compared with the mice that received the control diet (Figure 7B). Although combined treatment with GTPs and BSp showed a striking effect of repression of breast tumor growth (inhibition rate $93.87 \%$ ), the ability of this combinatorial treatment on inhibiting of breast cancer initiation is the same as GTPs treatment alone (prevention rate 60\%) as interpreted in Table 2, indicating this dietary regimen is more efficacious in inhibiting early transformed breast cancer than precancerous breast lesions.

To further analyze the potential in vivo antiproliferative properties of dietary GTPs and BSp administration, we performed immunohistochemical assays to detect PCNA-positive cells as an in vivo indicator for cellular proliferation in mice SHR xenograft tumors. As shown in Figure 7C \& D, PCNA-positive cells that represent proliferating cells in mice xenograft tumors were significantly depleted by GTPs and BSp treatment alone $(\mathrm{p}<0.05)$ and the percentage of PCNA-positive cells were significantly reduced by 26.74-fold in breast tumors of the combination group compared with the control group $(\mathrm{p}<0.01)$. In addition, combinatorial treatment with GTPs and BSp further significantly inhibited PCNA expression ( $\mathrm{p}<$ 0.01 ) than the other single-treatment groups suggesting this combinatorial region induced a synergistic effect of cellular proliferation inhibition in vivo. This result indicates that dietary GTPs and BSp can inhibit breast tumor growth in an in vivo system by affecting the proliferation rate of xenograft tumor growth.

\section{Discussion}

The intriguing effects of the bioactive botanic components on cancer chemoprevention and therapy have recently received considerable attention due to their characteristics of safe use, physiological availability and robust control on multiple signal pathways that affect carcinogenesis. GTP EGCG and BSp SFN are among the most important bioactive dietary compounds that have potent anticancer properties [11-15]. Although various molecular mechanisms have been proposed involving EGCG- or SFN-induced inhibitory effects on many types of cancers such as induction of apoptosis and cell cycle arrest, another important mechanism modulated by EGCG and SFN pertains to influencing epigenetic pathways leading to correction of aberrant epigenetic marks in cancer cells [16-21]. Our previous studies and others have found that GTP EGCG can influence epigenetic pathway via direct and/or indirect inhibition of DNMTs activity, thereby leading to beneficial reversals of aberrant DNA methylation profiles of multiple tumor-related genes $[14,20]$. BSp SFN in cancer chemoprevention has recently surged due to its HDACs inhibition activity which leads to an increase in the global and local histone acetylation of a number of genes [15,21]. Although the mechanisms involved in EGCG- or SFN-induced anticancer properties have been intensively investigated, relatively few studies have focused on the combined potential of nutritive components and their epigenetic mechanisms. These botanicals have similar favorable biological effects on epigenetic regulation but different mechanisms for carrying out these effects. For example, EGCG acts as a DNMTs inhibitor reducing the aberrant gene hypermethylation and SFN is an HDACs inhibitor that can remodel chromatin structure by affecting histone acetylation status in cancer cells. Both compounds are effective when acting alone in preventing breast cancer in vitro and in vivo and their combined effects may be synergistic.

We therefore tested our hypothesis in vitro and in vivo to better understand the potential combinatorial effects of GTP EGCG and BSp SFN during early breast tumorigenesis, and more importantly, to explore the preventive or therapeutic properties of this combination and potential molecular mechanisms in reversing breast malignancy. We started our work on an established cancer chemoprevention model that causes normal breast cells to undergo cancer initiation. This cellular model system resembles both pathological progression as well as potential molecular events in early breast oncogenic transformation through sequential oncogenic transfection to normal human epithelial cells [26,27]. Although this is an artificial cellular system and the individual genetic mutations and subsequent alterations of signal pathways may not occur in all types of breast cancer, this model has been well documented to study potential molecular mechanisms during early breast tumorigenesis not only because it closely approximates the initiation and early progression of breast cancer, it also very easy to manipulate as stable transfected cell lines such as precancerous $\mathrm{SH}$ cells and transformed breast cancer SHR cells that represent different stages 
(A)

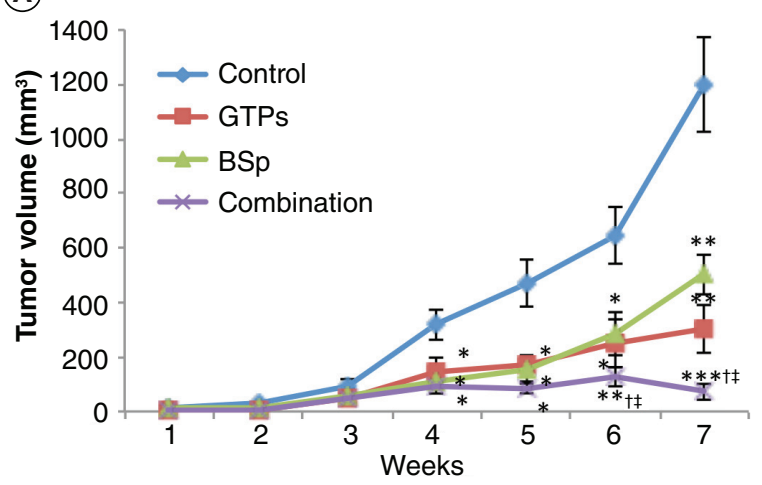

(C)
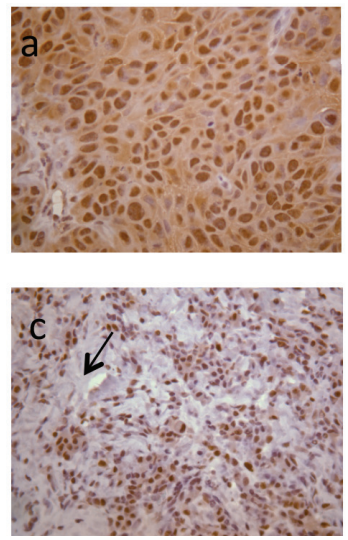
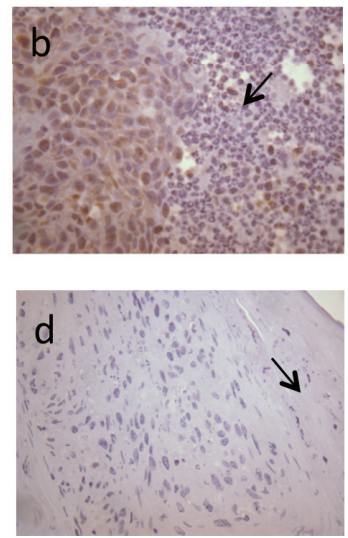

(B)

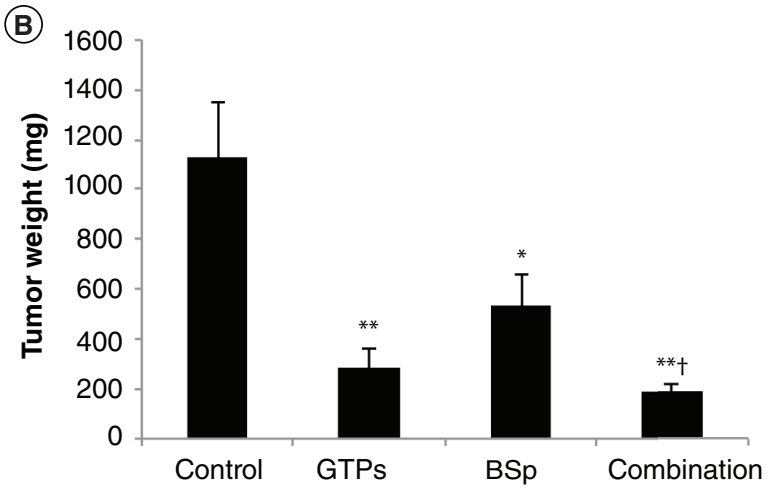

(D)

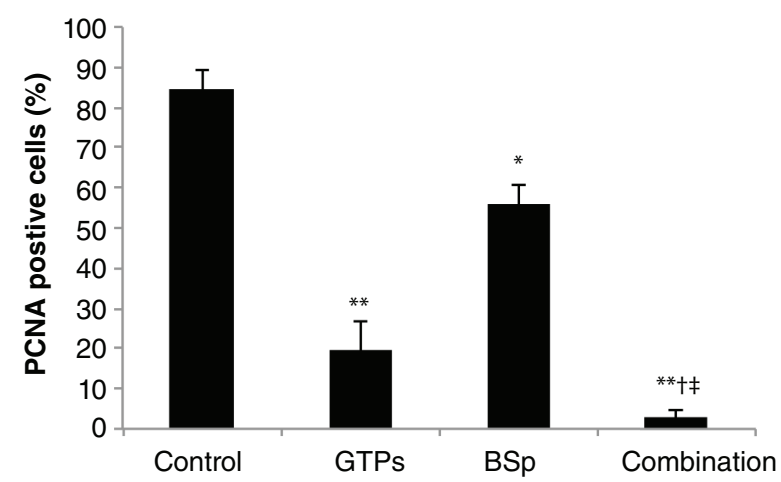

Figure 7. Inhibitory effects of GTPs and BSp diet on tumor growth of SHR breast cancer xenografts. Female athymic nu/nu mice were injected with $2 \times 10^{6}$ SHR cells and randomly separated to four groups (control, GTPs, BSp and combination group, see 'Materials and methods' section) with five mice in each group. Mice were administered either regular control diet, $0.5 \%$ GTPs in drinking water, $26 \%$ BSp diet or two diets in combination 2 weeks prior to injection and thereafter. (A) Tumor volume. Tumor volumes were observed weekly after injection and represented as mean values for each group. (B) Tumor weight. (C) Immunohistochemical analysis was performed in tumor samples to detect PCNA-positive cells for proliferation index (a, control; b, GTPs; c, BSp; d, combination group). Treatment with GTPs or BSp can cause local inflammation reaction and necrosis (see arrow point in b, c and d) in SHR breast xenografts. Representative photographs are from one field of each experimental group. (D) Bar chart is presented the immunohistochemical data in terms of percentage of PCNA-positive cells. PCNA-positive cells were counted in five different areas of the sections. Symbols and columns, mean; bars, standard deviation.

${ }^{\dagger} p<0.01$, significantly different from BSp group.

${ }^{\ddagger} \mathrm{p}<0.05$, significantly different from GTPs group.

${ }^{*} p<0.05,{ }^{* *} p<0.01, * * * p<0.001$, significantly different from control group.

BSp: Broccoli sprout; GTP: Green tea polyphenol; PCNA: Proliferating cell nuclear antigen.

of breast cancer are available [22-24]. This model therefore enables us to assess the impact of the botanical combinatorial regimen in real-time not only in preventing the transition to oncogenesis, but also in preventing the epigenetic aberrations that are frequently associated with breast cancer. Such studies will help to elucidate the potential of applying this novel botanical combination in future clinical trials.

In the present study, our results showed a synergistic effect on inhibition of breast cancer growth when EGCG and SFN were used together at relatively low concentrations of $20 \mu \mathrm{M}$ EGCG and $10 \mu \mathrm{M}$ SFN without causing toxicity in normal control breast HMECs. This result indicates that the combinatorial botanical approach is more efficacious than when these botanicals are used singly and that this combination is safe. The dietary combinatorial regimen using relatively low concentrations of EGCG and SFN is physiologically accessible by daily consumption of GTPs and BSp, which are equal to drinking $2-3$ cups of green tea and consuming $266 \mathrm{~g}$ ( $\sim 4$ cups) BSp/per day by an adult human, respectively $[14,15,55,56]$. Thus it has an important bioavailability toward chemoprevention and therapy against breast cancer. Importantly, 
Table 2. Combined treatment with green tea polyphenol and broccoli sprout inhibited tumor growth in mouse SHR xenografts.

\begin{tabular}{|c|c|c|c|c|c|}
\hline Animal group & Diet and treatment & Prevention rate $^{\dagger}(\%)$ & $\mathrm{TV}^{\ddagger} \mathrm{mm}^{3}($ mean $\pm \mathrm{SD})$ & RTV $^{\S}$ (mean) & IR TI $^{\text {\% } \%)}$ \\
\hline Control & Normal AIN-93G diet & 0 & $253-2402(1201 \pm 807)$ & 60.06 & - \\
\hline GTPs & $\begin{array}{l}0.5 \% \text { GTPs (Sunphenon 90D) in } \\
\text { drinking water }\end{array}$ & 60 & $60-1100(302 \pm 445)$ & 15.08 & 74.89 \\
\hline BSp & $\begin{array}{l}26 \% \text { BSp diet ( } 26 \mathrm{~g} \text { broccoli } \\
\text { sprout } / 100 \mathrm{~g} \text { AIN } 93 \mathrm{G} \text { diet) }\end{array}$ & 40 & $10-1800(502 \pm 750)$ & 25.09 & 58.22 \\
\hline Combination & $\begin{array}{l}0.5 \% \text { Poly-E in drinking water } \\
\text { and } 26 \% \text { BSp diet }\end{array}$ & 60 & $0-188(74 \pm 101)$ & 3.68 & 93.87 \\
\hline \multicolumn{6}{|c|}{ 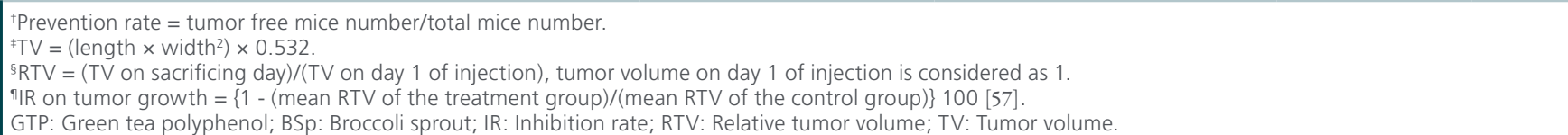 } \\
\hline
\end{tabular}

the combined use of GTPs and SFN in breast cancer prevention and therapy could potentially allow lower levels of consumption with no toxicity, which is crucial to overcome many of the barriers of combinatorial chemoprevention while significantly advancing the use of these dietary components.

This combination also induced significant cellular apoptosis and cell cycle arrest in S- and G2/M-phases in early transformed breast cancer SHR cells. Interestingly, we found that EGCG and SFN administration induced more significant effects on cellular viability inhibition, apoptotic response and cell cycle arrest in early transformed breast cancer SHR cells rather than precancerous $\mathrm{SH}$ cells. These results suggest that this dietary combination regimen may predominately employ its anticancer effect at early stages of breast tumorigenesis, indicating important chemotherapeutic and chemoprevention effects of this dietary regimen on inhibiting early breast progression that may apply in future clinical trials.

Further mechanistic studies revealed that the combinatorial treatment with EGCG and SFN may influence epigenetic pathways via inducing alterations of gene expression and enzymatic activities of DNMT1 and HDAC1 in early transformed breast cancer. Aberrant epigenetic modifications frequently occur during breast tumorigenesis. For example, aberrant DNA methylation status and abnormal histone modification profiles may lead to perturbations of chromatin structure, dysregulation of gene expression and genomic instability, that contribute to early cellular transformation and malignant initiation [1-5]. This abnormities are more prevalent in early transforming tumor cells, which could explain the phenomenon we found in our study that combined treatment with EGCG and SFN are more efficacious in early transformed breast SHR cells. Genome-wide methylation analysis showed differential DNA methylation status changes in multiple tumor-related genes in early transformed breast cancer cells in response to this combinatorial regimen. In addition, combination treatment with EGCG and SFN also significantly increased global acetylation level of histone $\mathrm{H} 3$. These results suggest that these dietary compounds may exert their anticancer effects primarily through reversing aberrant epigenetic codes during early breast tumorigenesis. Besides EGCG in green tea and SFN in broccoli sprouts, there are many epigenetic diets such as genistein in soybean and resveratrol in berries that have been identified to frequently regulate key tumor-related gene expression including ER $\alpha, h T E R T$ and $p 16^{I N K 4 a}$ via epigenetic mechanisms leading to various cancer chemoprevention effects [14-15,23,58-59]. Further results indicate causal effects of DNMT1 and HDAC1 gene expressions and/or enzymatic enzymatic activities on regulation of key tumor-related genes suggesting epigenetic mechanisms play a major role in this novel dietary combinatorial regimeninduced chemoprevention and therapeutic effect on early breast cancer.

We also tested this dietary regimen in a mouse xenograft system. Consistently, orally fed GTPs and BSp diets significantly inhibited tumor growth of breast cancer xenografts than any of these two diets acting alone. The bioavailability of EGCG is considered high compared with other tea catechins since a large proportion of EGCG in plasma is in a free form [55]. Studies also show that SFN is rapidly absorbed and displayed an absolute bioavailability of $82 \%$ in a dose-dependent pharmacokinetic behavior [60]. Thus, this combinatorial dietary regimen can achieve physiologically relevant bioavailability and practical consumption levels in vivo. Practically, this can also be achieved by consuming concentrated BSp/SFN supplement for future clinical trial. Collectively, these studies indicate that combined GTPs and BSp are highly effective in inhibiting early breast 
cancer development by, at least in part, regulating epigenetic mechanisms.

\section{Conclusion}

In summary, our findings provide important observations for the use of safe and effective combinations of epigenetic botanical compounds including GTP EGCG and BSp SFN to reverse epigenetic aberrations in early breast cancer. This novel dietary combinatorial regimen has high translational potential by providing lower doses of these compounds, increased practicality and compliance, reduced toxicity risk as well as enhanced efficacy and greater cost effectiveness in breast cancer management. Future efforts aimed at determining the appropriate administration of these combined botanicals in breast cancer patients and elucidating the further anticancer mechanisms are needed.

\section{Author contributions}

Y Li designed and conducted the experiments and drafted the manuscript. P Buckhaults assisted with some experiments and genome-wide DNA methylation analysis. XQ Cui assisted with genome-wide DNA methylation analysis. TO Tollefsbol conceived the study, contributed to the design of the experiments and revised the manuscript critically for important in- tellectual content. All authors read and approved the final manuscript.

Financial \& competing interests disclosure

This work was supported by grants from the National Cancer Institute (R01 CA178441; R03 CA176766) and the American Institute for Cancer Research (316184). The authors have no other relevant affiliations or financial involvement with any organization or entity with a financial interest in or financial conflict with the subject matter or materials discussed in the manuscript apart from those disclosed.

No writing assistance was utilized in the production of this manuscript.

Ethical conduct of research

The authors state that they have obtained appropriate institutional review board approval or have followed the principles outlined in the Declaration of Helsinki for all human or animal experimental investigations. In addition, for investigations involving human subjects, informed consent has been obtained from the participants involved.

\section{Open access}

This work is licensed under the Attribution-NonCommercialNoDerivatives 4.0 Unported License. To view a copy of this license, visit http://creativecommons.org/licenses/by-nc-nd/4.0/

\section{Executive summary}

Aim

- Breast cancer is the most common cancer and the second leading cause of cancer death among women.

- Aberrant epigenetic events are important contributors to the pathogenesis of breast cancer and dietary botanicals with properties affecting epigenetic processes are believed to influence early cancer development leading to cancer prevention effects.

- Previous studies indicate that singly administered bioactive dietary components with inhibitory properties on DNMTs and histone deacetylases, such as green tea polyphenols (GTPs) and broccoli sprouts (BSp), respectively, have potent anticancer effects both in vitro and in vivo.

- We therefore sought to investigate potential combinatorial effects of GTPs and BSp on neutralizing epigenetic aberrations in an established breast cancer transformation system.

Results

- Epigallocatechin-3-gallate (EGCG) in GTPs and sulforaphane (SFN) in BSp in combination significantly inhibited initiation of breast tumorigenesis.

- Combined treatment with EGCG and SFN-induced cellular apoptosis and cell cycle arrest in early transformed breast cancer SHR cells.

- Gene expression and enzymatic activities of DNA methyltransferase 1 and histone deacetylase 1 may contribute to epigenetic regulation of key tumor-related genes leading to early breast cancer prevention and therapy.

- Combined treatment with EGCG and SFN leads to global changes in histone H3 acetylation and DNA methylation profiles.

- Combinatorial treatment with EGCG and SFN caused expression changes in tumor-related genes due to regulation of epigenetic pathways.

- Dietary GTPs and BSp significantly inhibited the growth of breast cancer xenografts more than any of these diets acting alone.

\section{Conclusion}

- Combined GTPs and BSp are highly effective in inhibiting early breast cancer development both in vitro and in vivo by, at least in part, regulating epigenetic mechanisms.

- This novel dietary combinatorial regimen has high translational potential for breast cancer management. 


\section{References}

1 CDC. 1999-2012 Cancer Incidence and Mortality Data. www.nccd.cdc.gov

2 Byler S, Goldgar S, Heerboth S et al. Genetic and epigenetic aspects of breast cancer progression and therapy. Anticancer Res. 34, 1071-1077 (2014).

3 Huang Y, Nayak S, Jankowitz R, Davidson NE, Oesterreich $S$. Epigenetics in breast cancer: what's new? Breast cancer Res. 13,225 (2011).

4 Jovanovic J, Rønneberg JA, Tost J, Kristensen V. The epigenetics of breast cancer. Mol. Oncol. 4, 242-254 (2010).

5 Baylin SB, Ohm JE. Epigenetic gene silencing in cancer - a mechanism for early oncogenic pathway addiction? Nat. Rev. Cancer 6, 107-116 (2006).

6 Kouzarides T. Chromatin modifications and their function. Cell 128, 693-705 (2007)

7 Herman JG, Baylin SB. Gene silencing in cancer in association with promoter hypermethylation. N. Engl. J. Med. 349, 2042-2054 (2003).

8 Nephew KP, Huang TH. Epigenetic gene silencing in cancer initiation and progression. Cancer Lett. 190, 125-133 (2003).

9 Yoo CB, Jones PA. Epigenetic therapy of cancer: past, present and future. Nat. Rev. Drug Discov. 5, 37-50 (2006).

10 Szyf M. Epigenetics, DNA methylation, and chromatin modifying drugs. Annu. Rev. Pharmacol. Toxicol. 49, 243-263 (2009).

11 Meeran SM, Ahmed A, Tollefsbol TO. Epigenetic targets of bioactive dietary components for cancer prevention and therapy. Clin. Epigenetics 1, 101-116 (2010).

12 Li Y, Tollefsbol TO. Impact on DNA methylation in cancer prevention and therapy by bioactive dietary components. Curr. Med. Chem. 17, 2141-2151 (2010).

13 Hardy TM, Tollefsbol TO. Epigenetic diet: impact on the epigenome and cancer. Epigenomics 3, 503-518 (2011).

14 Li Y, Yuan YY, Meeran SM, Tollefsbol TO. Synergistic epigenetic reactivation of estrogen receptor- $\alpha(\mathrm{ER} \alpha)$ by combined green tea polyphenol and histone deacetylase inhibitor in ER $\alpha$-negative breast cancer cells. Mol. Cancer 9, 274 (2010).

15 Meeran SM, Patel SN, Tollefsbol TO. Sulforaphane causes epigenetic repression of hTERT expression in human breast cancer cell lines. PLoS ONE 5, e11457 (2010).

16 Yang C, Landau J, Huang M, Newmark H. Inhibition of carcinogenesis by dietary polyphenolic compounds. Annu. Rev. Nutr. 21, 381-406 (2001).

17 Thangapazham R, Singh A, Sharma A, Warren J, Gaddipati J, Maheshwari R. Green tea polyphenols and its constituent epigallocatechin gallate inhibits proliferation of human breast cancer cells in vitro and in vivo. Cancer Lett. 245, 232-241 (2007).

18 Pledgie-Tracy A, Sobolewski MD, Davidson NE. Sulforaphane induces cell type-specific apoptosis in human breast cancer cell lines. Mol. Cancer Ther. 6, 1013-1021 (2007).

19 Cheung KL, Kong AN. Molecular targets of dietary phenethyl isothiocyanate and sulforaphane for cancer chemoprevention. AAPS J. 12, 87-97 (2010).
20 Fang M, Wang Y, Ai N et al. Tea polyphenol (-)-epigallocatechin-3-gallate inhibits DNA methyltransferase and reactivates methylation-silenced genes in cancer cell lines. Cancer Res. 63, 7563-7570 (2003).

21 Ho E, Clarke JD, Dashwood RH. Dietary sulforaphane, a histone deacetylase inhibitor for cancer prevention. J. Nutr. 139, 2393-2396 (2009).

22 DeAngelis JT, Li Y, Mitchell N, Wilson L, Kim H, Tollefsbol TO. 2D difference gel electrophoresis analysis of different time points during the course of neoplastic transformation of human mammary epithelial cells. J. Proteome. Res. 10, 447-458 (2011).

23 Li Y, Chen H, Hardy TM, Tollefsbol TO. Epigenetic regulation of multiple tumor-related genes leads to suppression of breast tumorigenesis by dietary genistein. PLoS ONE 8, e54369 (2013).

24 Mitchell NE, Wilson ML, Bray MS, Crossman DK, Tollefsbol TO. Real-time methylomic aberrations during initiation and progression of induced human mammary epithelial cell tumorigenesis. Epigenomics 5, 155-165 (2013).

25 Power and Sample Size. http://powerandsamplesize.com

26 Hahn WC, Counter CM, Lundberg AS, Beijersbergen RL, Brooks MW. Creation of human tumour cells with defined genetic elements. Nature 400, 464-468 (1999).

27 Elenbaas B, Spirio L, Koerner F, Fleming MD, Zimonjic DB. Human breast cancer cells generated by oncogenic transformation of primary mammary epithelial cells. Genes Dev. 15, 50-65 (2001).

28 Chou TC. Theoretical basis, experimental design, and computerized stimulation of synergism and antagonism in drug combination studies. Pharmacol. Rev. 58, 621-681 (2006).

29 Ye L, Dinkova-Kostova AT, Wade KL, Zhang Y, Shapiro TA, Talalay P. Quantitative determination of dithiocarbamates in human plasma, serum, erythrocytes and urine: pharmacokinetics of broccoli sprout isothiocyanates in humans. Clin. Chim. Acta 316, 43-53 (2002).

30 Lee MJ, Maliakal P, Chen L et al. Pharmacokinetics of tea catechins after ingestion of green tea and (-)-epigallocatechin3-gallate by humans: formation of different metabolites and individual variability. Cancer Epidemiol. Biomarkers Prev. 11, 1025-1032 (2002)

31 Wu L, Griffin JD. Modulation of Notch signaling by mastermind-like (MAML) transcriptional co-activators and their involvement in tumorigenesis. Semin. Cancer Biol. 14, 348-356 (2004).

32 Stevens VL, Rodriguez C, Pavluck AL, Thun MJ, Calle EE. Association of polymorphisms in the paraoxonase 1 gene with breast cancer incidence in the CPS-II Nutrition Cohort. Cancer Epidemiol. Biomarkers Prev. 15, 1226-1228 (2006).

33 Magnani L, Ballantyne EB, Zhang X, Lupien M. PBX1 genomic pioneer function drives $\mathrm{ER} \alpha$ signaling underlying progression in breast cancer. PLoS Genet. 7, e1002368 (2011).

34 Gordon JA, Hassan MQ, Koss M et al. Epigenetic regulation of early osteogenesis and mineralized tissue formation by a HOXA10-PBX1-associated complex. Cells Tissues Organs 194, 146-150 (2011). 

polypeptides expressed in pancreatic cancer may serve as potential diagnostic markers and therapeutic targets for early stage adenocarcinomas. Pharm. Res. 30, 2260-2269 (2013)

37 Reubi JC, Schaer JC, Waser B. Cholecystokinin(CCK)-A and CCK-B/gastrin receptors in human tumors. Cancer Res. 57, 1377-1386 (1997). gene is amplified and overexpressed in mouse mammary gland adenocarcinomas and human breast cancer cell lines. Cancer Res. 63, 2179-2187 (2003). DNA is a sensitive and specific blood test for colorectal cancer. BMC Med. 9, 133 (2011).

40 Charalambous M, Smith FM, Bennett WR, Crew TE, Mackenzie F, Ward A. Disruption of the imprinted Grb10 gene leads to disproportionate overgrowth by an Igf2-independent mechanism. Proc. Natl Acad. Sci. USA 100, 8292-8297 (2003).

41 Jäkel S, Albig W, Kutay U et al. The importin beta/ importin 7 heterodimer is a functional nuclear import receptor for histone H1. EMBO J. 18, 2411-2423 (1999).

42 Guo S, Yang S, Taylor C, Sonenshein GE. Green tea polyphenol epigallocatechin-3 gallate (EGCG) affects gene expression of breast cancer cells transformed by the carcinogen 7,12-dimethylbenz[a] anthracene. J. Nutr. 135, 2978S-2986S (2005)

43 Jiang M, Gao Y, Yang T, Zhu X, Chen J. Cyclin Y: a novel membrane-associated cyclin, interacts with PFTK1. FEBS Lett. 583, 2171-2178 (2009).

44 Pasquale EB. Eph receptors and ephrins in cancer: bidirectional signalling and beyond. Nat. Rev. Cancer 10, 165-180 (2010).

45 Cammas L, Romand R, Fraulob V, Mura C, Dollé P. Expression of the murine retinol dehydrogenase 10 (Rdh10) gene correlates with many sites of retinoid signalling during embryogenesis and organ differentiation. Dev. Dyn. 236, 2899-2908 (2007).

46 Du H, Huang X, Wang S, Wu Y, Xu W, Li M. PSMA7, a potential biomarker of diseases. Protein. Pept. Lett. 16, 486-489 (2009).

47 Lahn M, Köhler G, Sundell K et al. Protein kinase C alpha expression in breast and ovarian cancer. Oncology 67, 1-10 (2004).

48 Chen C, Zhang Y, Zhang L, Weakley SM, Yao Q. MicroRNA-196: critical roles and clinical applications in development and cancer. J. Cell Mol. Med. 15, 14-23 (2011).
49 Liang J, Fu Y, Cruciat CM et al. Transmembrane protein 198 promotes LRP6 phosphorylation and Wnt signaling activation. Mol. Cell Biol. 31, 2577-2590 (2011).

50 Jezierska A, Olszewski WP, Pietruszkiewicz J, Olszewski W, Matysiak W, Motyl T. Activated Leukocyte Cell Adhesion Molecule (ALCAM) is associated with suppression of breast cancer cells invasion. Med. Sci. Monit. 12, BR245-BR256 (2006).

51 Kim M, Lee KT, Jang HR et al. Epigenetic down-regulation and suppressive role of $D C B L D 2$ in gastric cancer cell proliferation and invasion. Mol. Cancer Res. 6, 222-230 (2008).

52 Barbouti A, Höglund M, Johansson B et al. A novel gene, MSI2, encoding a putative RNA-binding protein is recurrently rearranged at disease progression of chronic myeloid leukemia and forms a fusion gene with HOXA9 as a result of the cryptic $\mathrm{t}(7 ; 17)(\mathrm{p} 15 ; \mathrm{q} 23)$. Cancer Res. 63 , 1202-1206 (2003).

53 Luft F. The rise of a ribosomopathy and increased cancer risk. J. Mol. Med. (Berl). 88, 1-3 (2010).

54 Rodriguez V, Chen Y, Elkahloun A, Dutra A, Pak E, Chandrasekharappa S. Chromosome 8 BAC array comparative genomic hybridization and expression analysis identify amplification and overexpression of TRMT12 in breast cancer. Genes Chromosomes Cancer 46, 694-707 (2007)

55 Thakur VS, Gupta K, Gupta S. The chemopreventive and chemotherapeutic potentials of tea polyphenols. Curr. Pharm. Biotechnol. 13, 191-199 (2012).

56 Shen G, Khor TO, Hu R et al. Chemoprevention of familial adenomatous polyposis by natural dietary compounds sulforaphane and dibenzoylmethane alone and in combination in ApcMin/+ mouse. Cancer Res. 67, 9937 (2007)

57 Utsugi T, Shibata J, Sugimoto Y et al. Antitumor activity of a novel podophyllotoxin derivative (TOP-53) against lung cancer and lung metastatic cancer. Cancer Res. 56, 2809-2814 (1996).

58 Li Y, Meeran SM, Patel SN, Chen H, Hardy TM, Tollefsbol TO. Epigenetic reactivation of estrogen receptor- $\alpha(E R \alpha)$ by genistein enhances hormonal therapy sensitivity in ER $\alpha$ negative breast cancer. Mol. Cancer 12, 9-25 (2013).

59 Kala R, Tollefsbol T. A novel combinatorial epigenetic therapy using resveratrol and pterostilbene for restoring estrogen receptor- $\alpha(E R \alpha)$ expression in $E R \alpha$-negative breast cancer cells. PLoS ONE 11(5), e0155057 (2016).

60 Hanlon N, Coldham N, Gielbert A et al. Absolute bioavailability and dose-dependent pharmacokinetic behaviour of dietary doses of the chemopreventive isothiocyanate sulforaphane in rat. Br. J. Nutr. 99, 559-564 (2008). 